ESAIM: M2AN 47 (2013) 183-211

DOI: $10.1051 / \mathrm{m} 2 \mathrm{an} / 2012025$
ESAIM: Mathematical Modelling and Numerical Analysis

www.esaim-m2an.org

\title{
ERROR ESTIMATES FOR THE ULTRA WEAK VARIATIONAL FORMULATION IN LINEAR ELASTICITY*
}

\author{
Teemu Luostari ${ }^{1}$, Tomi Huttunen ${ }^{1}$ And Peter Monk ${ }^{2}$
}

\begin{abstract}
We prove error estimates for the ultra weak variational formulation (UWVF) in 3D linear elasticity. We show that the UWVF of Navier's equation can be derived as an upwind discontinuous Galerkin method. Using this observation, error estimates are investigated applying techniques from the theory of discontinuous Galerkin methods. In particular, we derive a basic error estimate for the UWVF in a discontinuous Galerkin type norm and then an error estimate in the $\mathrm{L}^{2}(\Omega)$ norm in terms of the best approximation error. Our final result is an $\mathrm{L}^{2}(\Omega)$ norm error estimate using approximation properties of plane waves to give an estimate for the order of convergence. Numerical examples are presented.
\end{abstract}

Mathematics Subject Classification. 65N15, 65N30, 74J05, 74S30.

Received January 6, 2012. Revised May 2, 2012.

Published online August 31, 2012.

\section{INTRODUCTION}

In this paper we shall provide an error analysis of a non-polynomial, volume-based, Trefftz-type method: the ultra weak variational formulation (UWVF) $[5,6]$ applied to the Navier equation in 3D linear elasticity. The original UWVF for the Helmholtz and Maxwell's equations was proposed in [5,6]. The UWVF for 2D linear elasticity was derived in [16] following the strategies from [5,6] and for the fluid-solid interface problem in [19]. We will propose and analyze a $3 \mathrm{D}$ version of the method in [16].

In $[5,6]$, Cessenat and Després proved convergence estimates for the acoustic and electromagnetic UWVF in the $\mathrm{L}^{2}$-norm on the boundary of the domain. Using techniques from the analysis of discontinuous Galerkin methods a global error estimate for the acoustic UWVF are provided in [4]. Furthermore, Hiptmair et al. [13,14, 26] have proved error estimates for the more general plane wave based discontinuous Galerkin method (DGM) of the Helmholtz equation [13,26] and Maxwell's equations [14], results that are also applicable to the UWVF. The error estimates in $[13,14,26]$ are derived using the approximation properties of plane waves. Recently,

\footnotetext{
Keywords and phrases. Ultra weak variational formulation, error estimates, plane wave basis, linear elasticity, upwind discontinuous Galerkin method.

* Authors acknowledge Finnish Doctoral Programme in Computational Sciences (FICS) and Academy of Finland (project 213476 Finnish Centre of Excellence in Inverse Problems Research). The research of P. Monk was partially supported by the grant FA9550-05-1-0127 from the US-AFOSR.

1 Department of Applied Physics, University of Eastern Finland, P.O. Box 1627, 70211 Kuopio, Finland.

teemu.luostari@uef.fi; tomi.huttunen@uef.fi

2 Department of Mathematical Sciences, University of Delaware, Newark, 19716 DE, USA. monk@math.udel.edu
} 
Moiola $[24,25]$ has proved best approximation estimates for plane wave approximation in linear elasticity using the approximation properties of the plane wave basis functions in acoustics [13] and electromagnetism [14]. Approximation properties of plane waves are also investigated for the partition unity finite element method (PUFEM) [23], and the least-square and collocation formulations [29]. The PUFEM has also been used in 2D elasticity, see, for example, [8].

In addition to the UWVF, DGM and PUFEM, other non-polynomial methods that can be applied to linear elasticity problems include, for example, the least-squares method (LSM) [27] and the non-polynomial finite element method [2]. To date, the LSM and non-polynomial finite element method have been used in 2D acoustic problems. In 3D, the discontinuous enrichment method (DEM) [9, 10], a Lagrange multiplier type discontinuous Galerkin method has been applied to various wave modeling problems successfully including fluid-solid problems [22]. Recently [31] has compared the DEM to the UWVF and PUFEM for acoustic problems.

In this paper we derive the UWVF of the Navier's equation and use the discontinuous Galerkin scheme as a tool for error analysis, cf. [4,11,18]. Since this work is a continuation of [16], we borrow some estimates from it and extend the error estimates/analysis of the elastic UWVF. The analysis of the basic discontinuous Galerkin type error estimates and the error estimates in $L^{2}(\Omega)$ (without using the approximation properties of plane waves) follow mainly the strategies from $[4-7,27]$. In particular, when proving the discontinuous Galerkin type error estimate we are guided by [4]. However, we need to make several modifications to deal with vector and matrix quantities in the derivations, including in the definitions of jumps and averages. In addition, in the 3D elasticity the numerical flux is a $3 \times 3$ matrix and the boundary conditions are given in vector form. The analysis of the error estimate in $\mathrm{L}^{2}(\Omega)$ is based on a duality technique introduced in [27] and regularity estimates for linear elasticity [7]. The final error analysis of the UWVF in this paper is mainly motivated by the work of Hiptmair and coworkers $[13,14,24-26]$. In particular, we use the approximation properties of plane wave basis functions in linear elasticity to obtain our main convergence result. In [24,25] the approximation properties of plane waves are proved assuming that the number of basis functions for P- and S-waves are the same. However, we proceed very slightly differently to $[24,25]$ to obtain our main convergence result taking into account the fact that in practice the number of basis functions for P- and S-waves can be different. We shall also present some preliminary numerical results for a simple model problem. We consider the time-harmonic elastic plane wave propagation in a 3D unit cube with a Robin boundary condition, $c f .[16,21]$.

This paper is organized as follows. First we derive the UWVF of Navier's equation as a discontinuous Galerkin scheme and discuss the plane wave basis functions in 3D elasticity. Second we write the following error estimates: a basic discontinuous Galerkin type error estimate for the UWVF, an error estimate in $\mathrm{L}^{2}(\Omega)$ and the final error estimate using the approximation properties of plane wave basis functions. Third we show numerical results for $p$ - and $h$-convergence using uniform meshes. Note that preliminary 3D elastic UWVF results of $p$-convergence can be found in the conference paper [21]. Finally we draw some conclusions.

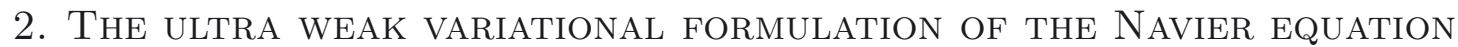

In this section we exhibit the connection between the ultra weak variational formulation (UWVF) for the Navier equation with the Robin boundary condition and an upwind discontinuous Galerkin method. The strategy of deriving the UWVF as a DG scheme is motivated by the acoustic case [4]. We shall also describe the plane wave basis functions used in 3D linear elasticity.

\subsection{The Navier equation}

Let $\Omega \subset \mathbb{R}^{3}$ be the computational domain, assumed to be a Lipschitz polyhedron (further restrictions will be placed on $\Omega$ as we develop the theory), and let $\Gamma$ denote its boundary. The lossless Navier problem that we shall consider is to find the time-harmonic displacement vector $\boldsymbol{u}$ such that

$$
\begin{array}{rc}
\mu \Delta \boldsymbol{u}+(\lambda+\mu) \nabla(\nabla \cdot \boldsymbol{u})+\omega^{2} \rho \boldsymbol{u}=\mathbf{0} & \text { in } \Omega, \\
\boldsymbol{T}^{(\boldsymbol{n})}(\boldsymbol{u})-i \eta \boldsymbol{u}=Q\left(-\boldsymbol{T}^{(\boldsymbol{n})}(\boldsymbol{u})-i \eta \boldsymbol{u}\right)+\boldsymbol{g} & \text { on } \Gamma,
\end{array}
$$


where $\boldsymbol{T}^{(\boldsymbol{n})}(\boldsymbol{u})=2 \mu \frac{\partial \boldsymbol{u}}{\partial \boldsymbol{n}}+\lambda \boldsymbol{n} \nabla \cdot \boldsymbol{u}+\mu \boldsymbol{n} \times \nabla \times \boldsymbol{u}$ is the traction operator, $\boldsymbol{g}$ is the source term, $\boldsymbol{n}$ is the outward unit normal and $\eta$ is a numerical flux ( $\eta$ is a $3 \times 3$ matrix). The parameter $Q \in \mathbb{C},|Q| \leq 1$, is used to set the boundary condition. The angular frequency of the field is $\omega, \rho$ is the density of the medium and $\lambda$ and $\mu$ are the Lamé constants defined by

$$
\mu=\frac{E}{2(1-\nu)} \quad \text { and } \quad \lambda=\frac{E \nu}{(1+\nu)(1-2 \nu)},
$$

where $E$ is the Young's modulus and $\nu$ is the Poisson ratio of the solid. All these constants are assumed to be real and positive. The wave speeds for pressure wave (P-wave) and shear waves (S-waves) are

$$
c_{P}=\sqrt{\frac{\lambda+2 \mu}{\rho}} \text { and } c_{S}=\sqrt{\frac{\mu}{\rho}},
$$

respectively, and the wave numbers for $\mathrm{P}$-wave and $\mathrm{S}$-waves are

$$
\kappa_{P}=\frac{\omega}{c_{P}} \quad \text { and } \quad \kappa_{S}=\frac{\omega}{c_{S}} .
$$

\subsection{The UWVF for the Navier equation}

From now on we consider the Robin type boundary condition, i.e. $Q=0$ in (2.2). Our theory cannot handle $|Q|=1$ but it is applicable for any $|Q|<1$.

Let us recall the strain tensor

$$
\epsilon(\boldsymbol{u})=\frac{1}{2}\left(\nabla \boldsymbol{u}+(\nabla \boldsymbol{u})^{T}\right)
$$

where the superscript $T$ indicates transpose. The stress tensor is $\sigma(\boldsymbol{u})=(\lambda \nabla \cdot \boldsymbol{u}) I_{d}+2 \mu \epsilon(\boldsymbol{u})$ where $I_{d}$ is the identity matrix. The strain and the stress tensors are symmetric. Then the following connection between the traction operator $\boldsymbol{T}^{(\boldsymbol{n})}(\boldsymbol{u})$ and the stress tensor $\sigma(\boldsymbol{u})$ can be written

$$
\boldsymbol{T}^{(\boldsymbol{n})}(\boldsymbol{u})=\sigma(\boldsymbol{u}) \boldsymbol{n} .
$$

Equations (2.1) and (2.2) can then be written as a first order system

$$
\begin{aligned}
\sigma(\boldsymbol{u})=(\lambda \nabla \cdot \boldsymbol{u}) I_{d}+2 \mu \epsilon(\boldsymbol{u}) & \text { in } \Omega, \\
\nabla \cdot \sigma(\boldsymbol{u})+\omega^{2} \rho \boldsymbol{u}=\mathbf{0} & \text { in } \Omega, \\
\sigma(\boldsymbol{u}) \boldsymbol{n}-i \eta \boldsymbol{u}=\boldsymbol{g} & \text { on } \Gamma .
\end{aligned}
$$

Let the domain $\Omega$ be covered by non-overlapping elements $K_{k}$ i.e. $\Omega=\cup_{k=1}^{N} K_{k}$ where $N$ is the number of elements. In this paper we assume a mesh of regular tetraheda but hexahedra or other element shapes could also be considered. The boundary of element $K_{k}$ is denoted by $\partial K_{k}$.

We now need to use Betti's third identity, see, for example, $[16,20,28]$, that can be written, for sufficiently smooth vector functions $\boldsymbol{u}$ and $\boldsymbol{\phi}$ as follows:

$$
\int_{\partial K_{k}}[\sigma(\boldsymbol{u}) \boldsymbol{n} \cdot \overline{\boldsymbol{\phi}}-\sigma(\overline{\boldsymbol{\phi}}) \boldsymbol{n} \cdot \boldsymbol{u}] \mathrm{d} S=\int_{K_{k}}[\nabla \cdot \sigma(\boldsymbol{u}) \cdot \overline{\boldsymbol{\phi}}-\nabla \cdot \sigma(\overline{\boldsymbol{\phi}}) \cdot \boldsymbol{u}] \mathrm{d} V,
$$

where $\bar{\phi}$ denote the complex conjugate of $\phi$. If we assume that $\boldsymbol{u}$ and the smooth test function $\bar{\phi}$ satisfy equations (2.3)-(2.4) the volume integral in Betti's formula (2.6) vanishes. Hence,

$$
\int_{\partial K_{k}}[\sigma(\overline{\boldsymbol{\phi}}) \boldsymbol{n} \cdot \boldsymbol{u}-\sigma(\boldsymbol{u}) \boldsymbol{n} \cdot \overline{\boldsymbol{\phi}}] \mathrm{d} S=0 .
$$


We shall develop a scheme based on piecewise smooth solutions of (2.1) so we need to allow for discontinuities across element interfaces. For the numerical scheme, we replace $\boldsymbol{u}$ by a consistent numerical flux $\hat{\boldsymbol{u}}$ and $\sigma(\boldsymbol{u})$ by $\hat{\sigma}(\boldsymbol{u})$ on the faces of the mesh that we shall detail shortly. Then, on each element, we impose

$$
\int_{\partial K_{k}}(\hat{\boldsymbol{u}} \cdot \sigma(\bar{\phi}) \boldsymbol{n}-\hat{\sigma}(\boldsymbol{u}) \boldsymbol{n} \cdot \overline{\boldsymbol{\phi}}) \mathrm{d} S=0 .
$$

Let us consider first interior interfaces (and after this we shall investigate the boundary faces separately). Motivated by [1], we define the averages and jumps across a common face between the neighboring elements $K_{k}$ and $K_{j}$ for the elasticity problem by

$$
\begin{aligned}
\left\{\{\boldsymbol{u}\}=\frac{\boldsymbol{u}_{k}+\boldsymbol{u}_{j}}{2},\right. & \left\{[\sigma(\boldsymbol{u})\}=\frac{\sigma\left(\boldsymbol{u}_{k}\right)+\sigma\left(\boldsymbol{u}_{j}\right)}{2},\right. \\
\llbracket \boldsymbol{u} \rrbracket=\boldsymbol{u}_{k} \otimes \boldsymbol{n}_{k}+\boldsymbol{u}_{j} \otimes \boldsymbol{n}_{j}, & \llbracket \sigma(\boldsymbol{u}) \rrbracket=\sigma\left(\boldsymbol{u}_{k}\right) \boldsymbol{n}_{k}+\sigma\left(\boldsymbol{u}_{j}\right) \boldsymbol{n}_{j},
\end{aligned}
$$

where $\boldsymbol{n}_{k}$ is the unit outward normal to $K_{k}\left(\right.$ similarly $\left.\boldsymbol{n}_{j}\right), \boldsymbol{u}_{k}=\left.\boldsymbol{u}\right|_{K_{k}}, \boldsymbol{u}_{j}=\left.\boldsymbol{u}\right|_{K_{j}}$ and $\boldsymbol{a} \otimes \boldsymbol{b}=\boldsymbol{a} \boldsymbol{b}^{T}$ where $\boldsymbol{a}$ and $\boldsymbol{b}$ are column vectors. The flux functions, can then be defined face by face as

$$
\begin{aligned}
\hat{\boldsymbol{u}} & =\alpha\{\{\boldsymbol{u}\}]+\beta \llbracket \sigma(\boldsymbol{u}) \rrbracket, \\
\hat{\sigma}(\boldsymbol{u}) & =\gamma\{\{\sigma(\boldsymbol{u})\}+\delta \llbracket \boldsymbol{u} \rrbracket,
\end{aligned}
$$

where the parameters $\alpha, \beta, \gamma$ and $\delta$ are yet to be chosen. Consistency requires that $\alpha=\gamma=1$ (so that, for example, if $\boldsymbol{u}$ is a solution of (2.3)-(2.4) on $K_{k} \cup K_{j}$ then $\boldsymbol{u}=\hat{\boldsymbol{u}}$ on their common face). The remaining coefficients are chosen so that we have equivalence between an upwind DG method and the UWVF scheme. We need to choose $\beta=-i \eta^{-1} / 2, \delta=i \eta / 2$ so that

$$
\begin{aligned}
\hat{\boldsymbol{u}} & =\left\{\{\boldsymbol{u}\}-\frac{i \eta^{-1}}{2} \llbracket \sigma(\boldsymbol{u}) \rrbracket,\right. \\
\hat{\sigma}(\boldsymbol{u}) & =\left\{\{\sigma(\boldsymbol{u})\}+\frac{i \eta}{2} \llbracket \boldsymbol{u} \rrbracket,\right.
\end{aligned}
$$

where $\eta$ is a real positive definite numerical flux matrix on each face (to be detailed in Sect. 4.1). To see the equivalence between the UWVF and upwind DG schemes let us multiply equation (2.7) by $i$ and plug equations (2.9) and (2.10) into equation (2.7). If $\Sigma_{k, j}$ denotes the common face between $K_{k}$ and $K_{j}$ (assuming $\left.\Sigma_{k, j} \neq \emptyset\right)$ with normal $\boldsymbol{n}_{k}$, we have

$$
\begin{aligned}
& \int_{\sum_{k, j}} i\left[\sigma\left(\bar{\phi}_{k}\right) \boldsymbol{n}_{k} \cdot \hat{\boldsymbol{u}}_{k}-\hat{\sigma}\left(\boldsymbol{u}_{k}\right) \boldsymbol{n}_{k} \cdot \overline{\boldsymbol{\phi}}_{k}\right] \mathrm{d} S=\int_{\sum_{k, j}} i\left[\frac{1}{2}\left(\boldsymbol{u}_{k}+\boldsymbol{u}_{j}\right)-\frac{i \eta^{-1}}{2}\left(\sigma\left(\boldsymbol{u}_{k}\right) \boldsymbol{n}_{k}+\sigma\left(\boldsymbol{u}_{j}\right) \boldsymbol{n}_{j}\right)\right] \cdot \sigma\left(\overline{\boldsymbol{\phi}}_{k}\right) \boldsymbol{n}_{k} \mathrm{~d} S \\
& -\int_{\sum_{k, j}} i\left[\frac{1}{2}\left(\sigma\left(\boldsymbol{u}_{k}\right)+\sigma\left(\boldsymbol{u}_{j}\right)\right) \boldsymbol{n}_{k}+\frac{i \eta}{2}\left(\boldsymbol{u}_{k} \otimes \boldsymbol{n}_{k}+\boldsymbol{u}_{j} \otimes \boldsymbol{n}_{j}\right) \boldsymbol{n}_{k}\right] \cdot \bar{\phi}_{k} \mathrm{~d} S .
\end{aligned}
$$

Note that

$$
\left(\boldsymbol{u}_{k} \otimes \boldsymbol{n}_{k}+\boldsymbol{u}_{j} \otimes \boldsymbol{n}_{j}\right) \boldsymbol{n}_{k}=\boldsymbol{u}_{k} \boldsymbol{n}_{k} \cdot \boldsymbol{n}_{k}+\boldsymbol{u}_{j} \boldsymbol{n}_{k} \cdot \boldsymbol{n}_{j}=\boldsymbol{u}_{k}-\boldsymbol{u}_{j} .
$$

Therefore, we can write

$$
\begin{aligned}
\int_{\sum_{k, j}} i\left[\sigma\left(\bar{\phi}_{k}\right) \boldsymbol{n}_{k} \cdot \hat{\boldsymbol{u}}_{k}-\hat{\sigma}\left(\boldsymbol{u}_{k}\right) \boldsymbol{n}_{k} \cdot \bar{\phi}_{k}\right] \mathrm{d} S= & \int_{\sum_{k, j}} i\left[\frac{1}{2}\left(\boldsymbol{u}_{k}+\boldsymbol{u}_{j}\right)-\frac{i \eta^{-1}}{2}\left(\sigma\left(\boldsymbol{u}_{k}\right) \boldsymbol{n}_{k}+\sigma\left(\boldsymbol{u}_{j}\right) \boldsymbol{n}_{j}\right)\right] \cdot \sigma\left(\bar{\phi}_{k}\right) \boldsymbol{n}_{k} \mathrm{~d} S \\
& -\int_{\sum_{k, j}} i\left[\frac{1}{2}\left(\sigma\left(\boldsymbol{u}_{k}\right)+\sigma\left(\boldsymbol{u}_{j}\right)\right) \boldsymbol{n}_{k}+\frac{i \eta}{2}\left(\boldsymbol{u}_{k}-\boldsymbol{u}_{j}\right)\right] \cdot \overline{\boldsymbol{\phi}}_{k} \mathrm{~d} S
\end{aligned}
$$


After more manipulation this becomes

$$
\begin{aligned}
\int_{\sum_{k, j}} i\left[\sigma\left(\overline{\boldsymbol{\phi}}_{k}\right) \boldsymbol{n}_{k} \cdot \hat{\boldsymbol{u}}_{k}-\hat{\sigma}\left(\boldsymbol{u}_{k}\right) \boldsymbol{n}_{k} \cdot \overline{\boldsymbol{\phi}}_{k}\right] \mathrm{d} S= & \int_{\sum_{k, j}} \frac{\eta^{-1}}{2}\left(-\sigma\left(\boldsymbol{u}_{k}\right) \boldsymbol{n}_{k}-i \eta \boldsymbol{u}_{k}\right) \cdot\left(-\sigma\left(\overline{\boldsymbol{\phi}}_{k}\right) \boldsymbol{n}_{k}+i \eta \overline{\boldsymbol{\phi}}_{k}\right) \mathrm{d} S \\
& -\int_{\sum_{k, j}} \frac{\eta^{-1}}{2}\left(-\sigma\left(\boldsymbol{u}_{j}\right) \boldsymbol{n}_{j}-i \eta \boldsymbol{u}_{j}\right) \cdot\left(\sigma\left(\overline{\boldsymbol{\phi}}_{k}\right) \boldsymbol{n}_{k}+i \eta \overline{\boldsymbol{\phi}}_{k}\right) \mathrm{d} S .
\end{aligned}
$$

Taking into account the complex conjugation we can rewrite equation (2.11) further as

$$
\begin{aligned}
\int_{\sum_{k, j}} i\left[\sigma\left(\bar{\phi}_{k}\right) \boldsymbol{n}_{k} \cdot \hat{\boldsymbol{u}}_{k}-\hat{\sigma}\left(\boldsymbol{u}_{k}\right) \boldsymbol{n}_{k} \cdot \overline{\boldsymbol{\phi}}_{k}\right] \mathrm{d} S= & \int_{\sum_{k, j}} \frac{\eta^{-1}}{2}\left(-\sigma\left(\boldsymbol{u}_{k}\right) \boldsymbol{n}_{k}-i \eta \boldsymbol{u}_{k}\right) \cdot \overline{\left(-\sigma\left(\phi_{k}\right) \boldsymbol{n}_{k}-i \eta \phi_{k}\right)} \mathrm{d} S \\
& -\int_{\sum_{k, j}} \frac{\eta^{-1}}{2}\left(-\sigma\left(\boldsymbol{u}_{j}\right) \boldsymbol{n}_{j}-i \eta \boldsymbol{u}_{j}\right) \cdot \overline{\left(\sigma\left(\phi_{k}\right) \boldsymbol{n}_{k}-i \eta \phi_{k}\right)} \mathrm{d} S .
\end{aligned}
$$

If we define $\mathcal{X}_{k}=\left(-\sigma\left(\boldsymbol{u}_{k}\right) \boldsymbol{n}_{k}-i \eta \boldsymbol{u}_{k}\right), \mathcal{Y}_{k}=\left(-\sigma\left(\phi_{k}\right) \boldsymbol{n}_{k}-i \eta \phi_{k}\right)$, and $F_{k}\left(\mathcal{Y}_{k}\right)=\left(\sigma\left(\phi_{k}\right) \boldsymbol{n}_{k}-i \eta \phi_{k}\right)$, then equation (2.12) can be written as

$$
\int_{\sum_{k, j}} i\left[\sigma\left(\bar{\phi}_{k}\right) \boldsymbol{n}_{k} \cdot \hat{\boldsymbol{u}}_{k}-\hat{\sigma}\left(\boldsymbol{u}_{k}\right) \boldsymbol{n}_{k} \cdot \overline{\boldsymbol{\phi}}_{k}\right] \mathrm{d} S=\frac{1}{2}\left[\int_{\sum_{k, j}} \eta^{-1} \mathcal{X}_{k} \cdot \overline{\mathcal{Y}}_{k} \mathrm{~d} S-\int_{\sum_{k, j}} \eta^{-1} \mathcal{X}_{j} \cdot \overline{F_{k}\left(\mathcal{Y}_{k}\right)} \mathrm{d} S\right] .
$$

Let us now consider an interior element $K_{k}$. Since (2.7) holds we can write, on the element,

$$
\int_{\partial K_{k}} \eta^{-1} \mathcal{X}_{k} \cdot \overline{\mathcal{Y}}_{k} \mathrm{~d} S-\sum_{j=1, j \neq k}^{N} \int_{\sum_{k, j}} \eta^{-1} \mathcal{X}_{j} \cdot \overline{F_{k}\left(\mathcal{Y}_{k}\right)} \mathrm{d} S=0
$$

where $N$ is the number of elements in the mesh (the second integral on the left hand side is computed only over the common faces between $K_{k}$ and its neighboring elements).

On the exterior boundary $\Gamma_{k}$ we define $\hat{\boldsymbol{u}}_{k}$ and $\hat{\sigma}\left(\boldsymbol{u}_{k}\right)$ in a special way compared to (2.9) and (2.10):

$$
\begin{aligned}
\hat{\boldsymbol{u}}_{k} & =\boldsymbol{u}_{k}, \\
\hat{\sigma}\left(\boldsymbol{u}_{k}\right) & =\sigma\left(\boldsymbol{u}_{k}\right) .
\end{aligned}
$$

Therefore, we can write on a boundary face $\Gamma_{k}=\Gamma \cap \partial K_{k}$,

$$
\int_{\Gamma_{k}} i\left[\sigma\left(\bar{\phi}_{k}\right) \boldsymbol{n}_{k} \cdot \hat{\boldsymbol{u}}_{k}-\hat{\sigma}\left(\boldsymbol{u}_{k}\right) \boldsymbol{n}_{k} \cdot \overline{\boldsymbol{\phi}}_{k}\right] \mathrm{d} S=\int_{\Gamma_{k}} i\left[\sigma\left(\overline{\boldsymbol{\phi}}_{k}\right) \boldsymbol{n}_{k} \cdot \boldsymbol{u}_{k}-\sigma\left(\boldsymbol{u}_{k}\right) \boldsymbol{n}_{k} \cdot \overline{\boldsymbol{\phi}}_{k}\right] \mathrm{d} S .
$$

Rearranging terms and taking $\eta^{-1}$ as a common factor we obtain

$$
\int_{\Gamma_{k}} i\left[\sigma\left(\bar{\phi}_{k}\right) \boldsymbol{n}_{k} \cdot \hat{\boldsymbol{u}}_{k}-\hat{\sigma}\left(\boldsymbol{u}_{k}\right) \boldsymbol{n}_{k} \cdot \overline{\boldsymbol{\phi}}_{k}\right] \mathrm{d} S=\int_{\Gamma_{k}} \eta^{-1}\left[-i \eta \sigma\left(\boldsymbol{u}_{k}\right) \boldsymbol{n}_{k} \cdot \overline{\boldsymbol{\phi}}_{k}+i \eta \sigma\left(\bar{\phi}_{k}\right) \boldsymbol{n}_{k} \cdot \boldsymbol{u}_{k}\right] \mathrm{d} S .
$$

Next we add and subtract terms $\sigma\left(\boldsymbol{u}_{k}\right) \boldsymbol{n}_{k} \cdot \sigma\left(\bar{\phi}_{k}\right) \boldsymbol{n}_{k} / 2$ and $\eta^{2} \boldsymbol{u}_{k} \cdot \bar{\phi}_{k} / 2$ and divide the above terms into two parts, and rearrange terms to get

$$
\begin{aligned}
\int_{\Gamma_{k}} i\left[\sigma\left(\overline{\boldsymbol{\phi}}_{k}\right) \boldsymbol{n}_{k} \cdot \hat{\boldsymbol{u}}_{k}-\hat{\sigma}\left(\boldsymbol{u}_{k}\right) \boldsymbol{n}_{k} \cdot \overline{\boldsymbol{\phi}}_{k}\right] \mathrm{d} S= & \int_{\Gamma_{k}} \frac{1}{2} \eta^{-1}\left(-\sigma\left(\boldsymbol{u}_{k}\right) \boldsymbol{n}_{k}-i \eta \boldsymbol{u}_{k}\right) \cdot \overline{\left(-\sigma\left(\phi_{k}\right) \boldsymbol{n}_{k}-i \eta \boldsymbol{\phi}_{k}\right)} \mathrm{d} S \\
& -\int_{\Gamma_{k}} \frac{1}{2} \eta^{-1}\left(\sigma\left(\boldsymbol{u}_{k}\right) \boldsymbol{n}_{k}-i \eta \boldsymbol{u}_{k}\right) \cdot \overline{\left(\sigma\left(\phi_{k}\right) \boldsymbol{n}_{k}-i \eta \phi_{k}\right)} \mathrm{d} S .
\end{aligned}
$$


Again, using the definitions of $\mathcal{X}_{k}, \mathcal{Y}_{k}, F_{k}\left(\mathcal{Y}_{k}\right)$ and defining a new function $F_{k}\left(\mathcal{X}_{k}\right)=\left(\sigma\left(\boldsymbol{u}_{k}\right) \boldsymbol{n}_{k}-i \eta \boldsymbol{u}_{k}\right)$ we obtain

$$
\int_{\Gamma_{k}} i\left[\sigma\left(\overline{\boldsymbol{\phi}}_{k}\right) \boldsymbol{n}_{k} \cdot \hat{\boldsymbol{u}}_{k}-\hat{\sigma}\left(\boldsymbol{u}_{k}\right) \boldsymbol{n}_{k} \cdot \overline{\boldsymbol{\phi}}_{k}\right] \mathrm{d} S=\frac{1}{2}\left[\int_{\Gamma_{k}} \eta^{-1} \mathcal{X}_{k} \cdot \overline{\mathcal{Y}}_{k} \mathrm{~d} S-\int_{\Gamma_{k}} \eta^{-1} F_{k}\left(\mathcal{X}_{k}\right) \cdot \overline{F_{k}\left(\mathcal{Y}_{k}\right)} \mathrm{d} S\right] .
$$

Taking into account the boundary condition (2.5), i.e.,

$$
\sigma(\boldsymbol{u}) \boldsymbol{n}-i \eta \boldsymbol{u}=\boldsymbol{g} \text { on } \Gamma,
$$

equation (2.16) can be written as

$$
\int_{\Gamma_{k}} i\left[\sigma\left(\bar{\phi}_{k}\right) \boldsymbol{n}_{k} \cdot \hat{\boldsymbol{u}}_{k}-\hat{\sigma}\left(\boldsymbol{u}_{k}\right) \boldsymbol{n}_{k} \cdot \overline{\boldsymbol{\phi}}_{k}\right] \mathrm{d} S=\frac{1}{2}\left[\int_{\Gamma_{k}} \eta^{-1} \mathcal{X}_{k} \cdot \overline{\mathcal{Y}}_{k} \mathrm{~d} S-\int_{\Gamma_{k}} \eta^{-1} \boldsymbol{g} \cdot \overline{F_{k}\left(\mathcal{Y}_{k}\right)} \mathrm{d} S\right] .
$$

Combining now equations (2.7), (2.13) and (2.17) we obtain

$$
\int_{\partial K_{k}} \eta^{-1} \mathcal{X}_{k} \cdot \overline{\mathcal{Y}}_{k} \mathrm{~d} S-\sum_{j=1, j \neq k}^{N} \int_{\Sigma_{k, j}} \eta^{-1} \mathcal{X}_{j} \cdot \overline{F_{k}\left(\mathcal{Y}_{k}\right)} \mathrm{d} S=\int_{\Gamma_{k}} \eta^{-1} \boldsymbol{g} \cdot \overline{F_{k}\left(\mathcal{Y}_{k}\right)} \mathrm{d} S .
$$

After summing over all elements, equation (2.18) gives

$$
\sum_{k=1}^{N}\left[\int_{\partial K_{k}} \eta^{-1} \mathcal{X}_{k} \cdot \overline{\mathcal{Y}}_{k} \mathrm{~d} S-\sum_{j=1, j \neq k}^{N} \int_{\Sigma_{k, j}} \eta^{-1} \mathcal{X}_{j} \cdot \overline{F_{k}\left(\mathcal{Y}_{k}\right)} \mathrm{d} S\right]=\sum_{k=1}^{N} \int_{\Gamma_{k}} \eta^{-1} \boldsymbol{g} \cdot \overline{F_{k}\left(\mathcal{Y}_{k}\right)} \mathrm{d} S .
$$

Motivated by [4], let us define the weighted space $\mathrm{L}_{\eta}^{2}\left(\partial K_{k}\right)$ using the weighted norm

$$
\|\boldsymbol{u}\|_{\mathrm{L}_{\eta}^{2}\left(\partial K_{k}\right)}^{2}=\int_{\partial K_{k}}\left(\eta^{-1} \boldsymbol{u}\right) \cdot \overline{\boldsymbol{u}} \mathrm{d} S
$$

where we note that both of our choices of $\eta$ in (4.1) and (4.2) are bounded and symmetric, positive definite matrix functions on the boundary of each element. Furthermore, let $X=\Pi_{K_{k} \in \mathcal{T}_{h}} \mathrm{~L}_{\eta}^{2}\left(\partial K_{k}\right)$ have the norm

$$
\|\mathcal{X}\|_{X}^{2}=\sum_{k=1}^{N} \int_{\partial K_{k}} \frac{1}{2}\left(\eta^{-1} \mathcal{X}_{k}\right) \cdot \overline{\mathcal{X}}_{k} \mathrm{~d} S=\frac{1}{2} \sum_{k=1}^{N}\left\|\mathcal{X}_{k}\right\|_{\mathrm{L}_{\eta}^{2}\left(\partial K_{k}\right)}^{2}
$$

and the inner product

$$
(\mathcal{X}, \mathcal{Y})_{X}=\sum_{k=1}^{N} \int_{\partial K_{k}} \frac{1}{2}\left(\eta^{-1} \mathcal{X}_{k}\right) \cdot \overline{\mathcal{Y}}_{k} \mathrm{~d} S .
$$

Motivated by (2.19) we define the sesquilinear form for the elastic UWVF by

$$
a(\mathcal{X}, \mathcal{Y})=\sum_{k=1}^{N} \int_{\partial K_{k}} \frac{1}{2} \eta^{-1} \mathcal{X}_{k} \cdot \overline{\mathcal{Y}}_{k} \mathrm{~d} S-\sum_{k=1}^{N} \sum_{j=1, j \neq k}^{N} \int_{\Sigma_{k, j}} \frac{1}{2} \eta^{-1} \mathcal{X}_{j} \cdot \overline{F_{k}\left(\mathcal{Y}_{k}\right)} \mathrm{d} S .
$$

The boundary data vector can be written as

$$
b(\mathcal{Y})=\sum_{k=1}^{N} \int_{\Gamma_{k}} \frac{1}{2} \eta^{-1} \boldsymbol{g} \cdot \overline{F_{k}\left(\mathcal{Y}_{k}\right)} \mathrm{d} S .
$$

Hence, we want to find $\mathcal{X} \in X$ so that

$$
a(\mathcal{X}, \mathcal{Y})=b(\mathcal{Y}) \quad \text { for all } \mathcal{Y} \in X
$$

This is the UWVF for the Navier equation (see [16]). 


\subsection{Basis functions and the discretization}

Next we define the elastic plane wave basis functions proceeding similarly to [16]. The solution of the adjoint Navier equation is separated into three components: P-wave (pressure wave), SH-wave (horizontal component of S-wave) and SV-wave (vertical component of S-wave). On each element $K_{k}$ we choose $p_{k}^{P}$ distinct unit vectors $\left\{\boldsymbol{a}_{k, \ell}^{P}\right\}, 1 \leq \ell \leq p_{k}^{P}$ and $p_{k}^{S}$ unit vectors $\left\{\boldsymbol{a}_{k, \ell}^{S}\right\}, 1 \leq \ell \leq p_{k}^{S}$. If $p_{k}^{P}=p_{k}^{S}$ these sets may be identical. These vectors define the direction of propagation of the plane waves that we now define by:

$$
\begin{aligned}
& \phi_{k, \ell}^{P}=\left\{\begin{array}{l}
\alpha_{k}^{P} \boldsymbol{a}_{k, \ell}^{P} \exp \left(i \bar{\kappa}_{P} \boldsymbol{a}_{k, \ell}^{P} \cdot \boldsymbol{x}\right) \text { in } K_{k} \\
0 \quad \text { elsewhere, }
\end{array}\right. \\
& \boldsymbol{\phi}_{k, \ell}^{S H}=\left\{\begin{array}{l}
\alpha_{k}^{S H} \boldsymbol{a}_{k, \ell}^{S \perp} \exp \left(i \bar{\kappa}_{S} \boldsymbol{a}_{k, \ell}^{S} \cdot \boldsymbol{x}\right) \text { in } K_{k} \\
0 \quad \text { elsewhere, }
\end{array}\right. \\
& \phi_{k, \ell}^{S V}=\left\{\begin{array}{l}
\alpha_{k}^{S V} \boldsymbol{a}_{k, \ell}^{S \perp} \times \boldsymbol{a}_{k, \ell}^{S} \exp \left(i \bar{\kappa}_{S} \boldsymbol{a}_{k, \ell}^{S} \cdot \boldsymbol{x}\right) \quad \text { in } K_{k} \\
0 \text { elsewhere, }
\end{array}\right.
\end{aligned}
$$

where $\boldsymbol{a}_{k, \ell}^{S \perp} \perp \boldsymbol{a}_{k, \ell}^{S}$ are unit vectors and $\alpha_{k}^{P}, \alpha_{k}^{S H}, \alpha_{k}^{S V}$ are the amplitudes. Note that, $\nabla \times \phi_{k}^{P}=0, \nabla \cdot \boldsymbol{\phi}_{k}^{S H}=$ $\nabla \cdot \phi_{k}^{S V}=0$ and $\boldsymbol{a}_{k, \ell}^{S} \perp \boldsymbol{a}_{k, \ell}^{S \perp} \perp\left(\boldsymbol{a}_{k, \ell}^{S \perp} \times \boldsymbol{a}_{k, \ell}^{S}\right)$.

The basis functions satisfy the adjoint Helmholtz equations on $K_{k}$ :

$$
\begin{aligned}
\Delta \overline{\boldsymbol{\phi}}_{k, \ell}^{P}+\kappa_{P}^{2} \overline{\boldsymbol{\phi}}_{k, \ell}^{P} & =\mathbf{0}, \\
\Delta \overline{\boldsymbol{\phi}}_{k, \ell}^{S H}+\kappa_{S}^{2} \overline{\boldsymbol{\phi}}_{k, \ell}^{S H} & =\mathbf{0}, \\
\Delta \overline{\boldsymbol{\phi}}_{k, \ell}^{S V}+\kappa_{S}^{2} \overline{\boldsymbol{\phi}}_{k, \ell}^{S V} & =\mathbf{0},
\end{aligned}
$$

respectively.

We can now define the subspaces used to construct the numerical scheme denoted $X_{k}^{h} \subset \mathrm{L}_{\eta}^{2}\left(\partial K_{k}\right), 1 \leq k \leq N$ and let $X^{h}=\Pi_{k=1}^{N} X_{k}^{h}$. Using the previously defined basis functions $\mathcal{X}_{k}^{h} \in X_{k}^{h}$ is given by

$$
\mathcal{X}_{k}^{h}=\sum_{\ell=1}^{p_{k}^{P}} \mathcal{X}_{k, \ell}^{P}\left(-\sigma\left(\phi_{k, \ell}^{P}\right) \boldsymbol{n}_{k}-i \eta \boldsymbol{\phi}_{k, \ell}^{P}\right)+\sum_{\ell=1}^{p_{k}^{S}} \mathcal{X}_{k, \ell}^{S H}\left(-\sigma\left(\boldsymbol{\phi}_{k, \ell}^{S H}\right) \boldsymbol{n}_{k}-i \eta \boldsymbol{\phi}_{k, \ell}^{S H}\right)+\sum_{\ell=1}^{p_{k}^{S}} \mathcal{X}_{k, \ell}^{S V}\left(-\sigma\left(\boldsymbol{\phi}_{k, \ell}^{S V}\right) \boldsymbol{n}_{k}-i \eta \boldsymbol{\phi}_{k, \ell}^{S V}\right)
$$

where $\left\{\mathcal{X}_{k, \ell}^{P}\right\}_{\ell=1}^{p_{k}^{P}},\left\{\mathcal{X}_{k, \ell}^{S H}\right\}_{\ell=1}^{p_{k}^{S}},\left\{\mathcal{X}_{k, \ell}^{S V}\right\}_{\ell=1}^{p_{k}^{S}}$ are expansion coefficients. Notice that the number of basis functions for $\mathrm{P}$ - and $\mathrm{S}$-waves can be different, and thus may be useful in practice to control ill-conditioning [16]. For $\mathcal{Y}_{k}^{h} \in X_{k}^{h}$, we have

$$
\mathcal{Y}_{k}^{h}=\sum_{\ell=1}^{p_{k}^{P}} \mathcal{Y}_{k, \ell}^{P}\left(-\sigma\left(\phi_{k, \ell}^{P}\right) \boldsymbol{n}_{k}-i \eta \phi_{k, \ell}^{P}\right)+\sum_{\ell=1}^{p_{k}^{S}} \mathcal{Y}_{k, \ell}^{S H}\left(-\sigma\left(\phi_{k, \ell}^{S H}\right) \boldsymbol{n}_{k}-i \eta \boldsymbol{\phi}_{k, \ell}^{S H}\right)+\sum_{\ell=1}^{p_{k}^{S}} \mathcal{Y}_{k, \ell}^{S V}\left(-\sigma\left(\boldsymbol{\phi}_{k, \ell}^{S V}\right) \boldsymbol{n}_{k}-i \eta \boldsymbol{\phi}_{k, \ell}^{S V}\right)
$$

for some expansion coefficients $\left\{\mathcal{Y}_{k, \ell}^{P}\right\}_{\ell=1}^{p_{k}^{P}},\left\{\mathcal{Y}_{k, \ell}^{S H}\right\}_{\ell=1}^{p_{k}^{S}},\left\{\mathcal{Y}_{k, \ell}^{S V}\right\}_{\ell=1}^{p_{k}^{S}}$. With this basis, $F_{k}\left(\mathcal{Y}_{k}^{h}\right)$ can easily computed via

$$
F\left(\mathcal{Y}_{k}^{h}\right)=\sum_{\ell=1}^{p_{k}^{P}} \mathcal{Y}_{k, \ell}^{P}\left(\sigma\left(\phi_{k, \ell}^{P}\right) \boldsymbol{n}_{k}-i \eta \phi_{k, \ell}^{P}\right)+\sum_{\ell=1}^{p_{k}^{S}} \mathcal{Y}_{k, \ell}^{S H}\left(\sigma\left(\phi_{k, \ell}^{S H}\right) \boldsymbol{n}_{k}-i \eta \phi_{k, \ell}^{S H}\right)+\sum_{\ell=1}^{p_{k}^{S}} \mathcal{Y}_{k, \ell}^{S V}\left(\sigma\left(\boldsymbol{\phi}_{k, \ell}^{S V}\right) \boldsymbol{n}_{k}-i \eta \boldsymbol{\phi}_{k, \ell}^{S V}\right) .
$$

We thus define

$$
\begin{aligned}
X_{k}^{h}= & \operatorname{span}\left\{\sigma\left(\boldsymbol{\phi}_{k, \ell}^{P}\right) \boldsymbol{n}_{k}+i \eta \boldsymbol{\phi}_{k, \ell}^{P}, \quad 1 \leq \ell \leq p_{k}^{P}, \quad \sigma\left(\boldsymbol{\phi}_{k, \ell}^{S H}\right) \boldsymbol{n}_{k}+i \eta \boldsymbol{\phi}_{k, \ell}^{S H}, \quad 1 \leq \ell \leq p_{k}^{S},\right. \\
& \left.\sigma\left(\boldsymbol{\phi}_{k, \ell}^{S V}\right) \boldsymbol{n}_{k}+i \eta \boldsymbol{\phi}_{k, \ell}^{S V}, \quad 1 \leq \ell \leq p_{k}^{S}\right\}
\end{aligned}
$$


for $k=1, \ldots, N$ and $X^{h}=\Pi_{k=1}^{N} X_{k}^{h}$. Hence, the discretized form of the problem (2.21), is to find $\mathcal{X}^{h} \in X^{h}$ so that

$$
a\left(\mathcal{X}^{h}, \mathcal{Y}^{h}\right)=b\left(\mathcal{Y}^{h}\right) \quad \text { for all } \mathcal{Y}^{h} \in X^{h}
$$

The UWVF can be written in the matrix system as

$$
(D-C) \boldsymbol{x}=\boldsymbol{b}
$$

where $\boldsymbol{x}=\left(x_{11}^{P}, \ldots, x_{1 p_{1}^{P}}^{P}, x_{11}^{S H}, \ldots, x_{1 p_{1}^{S}}^{S H}, x_{11}^{S V}, \ldots, x_{1 p_{1}^{S}}^{S V}, \ldots\right)^{T}, D$ is a diagonal, Hermitian, block matrix and the sparse matrix $C$ has at most 4 off-diagonal blocks on each block row when tetrahedral elements are used and $Q=0$. The entries of the matrix $D$ result from the first integral of equation (2.19) and matrix $C$ entries from the second integral $(Q=0)$, see more details in $[5,6,15,16]$. In [5] analytical formulas are provided to compute the integrals of the plane wave basis functions. Hence, the integrals in the sesquilinear form (2.20) can be computed in closed form. In practice, see [5,6], the linear system $(2.26)$ is solved using the preconditioned form:

$$
\left(I-D^{-1} C\right) \boldsymbol{x}=D^{-1} \boldsymbol{b}
$$

where $I$ is the identity matrix, and the matrix $D$ is inverted blockwise [15].

\section{ERror estimates}

In this section we derive error estimates adopting the approach from [4-6,13,14,16,24-26]. First we derive a basic error estimate in a discontinuous Galerkin type norm motivated by the results in [4]. Second we derive an error estimate with respect to the $\mathrm{L}^{2}(\Omega)$ norm. In particular, we use the regularity estimates for linear elasticity derived in [7] and use the dual problem approach from [27]. Finally, we derive the final $\mathrm{L}^{2}(\Omega)$ norm error estimate using the approximation properties of the plane wave basis functions applying strategies from $[13,14,24-26]$. In particular, we shall use the approach of [25] to obtain our final convergence result. Differing from [25] our final error estimate allows the numbers of basis functions for P- and S-waves to be different.

\subsection{Basic error estimate}

In this section we derive a basic discontinuous Galerkin type error estimate for the UWVF. This section is motivated by [4-6], but now applied to the elastic UWVF. Since this work is a continuation of the $2 \mathrm{D}$ elastic UWVF we also borrow some of the theoretical results from [16].

We recall the following important result that is easily extended to $3 \mathrm{D}$ :

Lemma 3.1 (Lem. 5.1 in [16]). Assume that the coefficients in the elastic UWVF are real and constant. The operator $F$ is an isometry so that $\|F\|_{X \rightarrow X}=1$, and $F$ is unitary i.e. $F^{*} F=I$ where $F^{*}$ is the adjoint of $F$ in the $X$-inner product.

Proof. The proof can be found in detail from [16]. The proof is based on the "Isometry Lemma", see Lemma 3.1 in [16] and the fact that all coefficients are real.

Following $[4,6,16]$, we define a new operator $\Pi: X \rightarrow X$ such that if $\mathcal{Y} \in X$ then

$$
\begin{aligned}
\left.\Pi \mathcal{Y}\right|_{\Sigma_{k, j}} & =\left.\mathcal{Y}\right|_{\Sigma_{j, k}}, \\
\left.\Pi \mathcal{Y}\right|_{\Gamma_{k}} & =0,
\end{aligned}
$$

for all $1 \leq k, j \leq N$ (note that $Q=0$ here). The following lemma gives relevant properties of the operator $\Pi$. 
Lemma 3.2 (Lem. 1.7 and Prop. 1.10 in [6]). The operator $\Pi: X \rightarrow X$ is bounded with

$$
\|\Pi\| \leq 1
$$

and for the induced norm of $F^{*} \Pi$ we have

$$
\left\|F^{*} \Pi\right\| \leq 1 .
$$

Proof. Only a sketch of the proof is represented here. The first claim $\|\Pi\|_{X} \leq 1$ follows from equations (3.1) and (3.2), and $\left\|F^{*} \Pi\right\|_{X} \leq 1$ follows from $\|\Pi\|_{X} \leq 1$ and the fact that the operator is a bijective isometry in $X$ [6], Lemma 1.12.

We now estimate $\left|a\left(\mathcal{X}-\mathcal{X}^{h}, \mathcal{X}-\mathcal{X}^{h}\right)\right|$, the consistency error of the elastic UWVF.

Theorem 3.3. Let $P^{h}: X \rightarrow X^{h}$ denote the best approximation projection in the $X$ norm. Then the following estimate holds

$$
\left|a\left(\mathcal{X}-\mathcal{X}^{h}, \mathcal{X}-\mathcal{X}^{h}\right)\right| \leq 2\left\|\left(I-P^{h}\right) \mathcal{X}^{h}\right\|_{X}^{2} .
$$

Proof. The proof is shown in [4] for the acoustic case, however, we outline it herein for the sake of completeness. Due to the Galerkin property we can write

$$
a\left(\mathcal{X}-\mathcal{X}^{h}, \mathcal{Y}^{h}\right)=0 \quad \text { for all } \mathcal{Y}^{h} \in X^{h} .
$$

Furthermore, because $P^{h}: X \rightarrow X^{h}$, we can write

$$
\begin{aligned}
a\left(\mathcal{X}-\mathcal{X}^{h}, \mathcal{X}-\mathcal{X}^{h}\right) & =a\left(\mathcal{X}-\mathcal{X}^{h},\left(I-P^{h}\right) \mathcal{X}\right)+a\left(\mathcal{X}-\mathcal{X}^{h}, P^{h} \mathcal{X}-\mathcal{X}^{h}\right) \\
& =a\left(\mathcal{X}-\mathcal{X}^{h},\left(I-P^{h}\right) \mathcal{X}\right) .
\end{aligned}
$$

For all $\mathcal{X}, \mathcal{Y} \in X$, equation $(2.20)$ can be rewritten into the form

$$
a(\mathcal{X}, \mathcal{Y})=(\mathcal{X}, \mathcal{Y})_{X}-(\Pi \mathcal{X}, F(\mathcal{Y}))_{X}=\left(\mathcal{X}-F^{*} \Pi \mathcal{X}, \mathcal{Y}\right)_{X}
$$

Applying (3.5) to $|a(\mathcal{X}, \mathcal{Y})|$ and the Cauchy-Schwarz inequality, we have the estimate

$$
|a(\mathcal{X}, \mathcal{Y})|=\left|\left(\left(I-F^{*} \Pi\right) \mathcal{X}, \mathcal{Y}\right)_{X}\right| \leq\left\|\left(I-F^{*} \Pi\right) \mathcal{X}\right\|_{X}\|\mathcal{Y}\|_{X} .
$$

Using Lemma 3.2, (see also [6], Prop. 1.10), we can write, (see [6], proof of Lemma 3.1),

$$
\left\|\left(I-F^{*} \Pi\right)\left(\mathcal{X}-\mathcal{X}^{h}\right)\right\|_{X} \leq 2\left\|\left(I-P^{h}\right) \mathcal{X}\right\|_{X} .
$$

Replacing $\mathcal{Y}$ by $\mathcal{X}-\mathcal{X}^{h}$ and $\mathcal{X}$ by $\mathcal{X}-\mathcal{X}^{h}$ in (3.6) and using the relation (3.4), we have

$$
\begin{aligned}
\left|a\left(\mathcal{X}-\mathcal{X}^{h}, \mathcal{X}-\mathcal{X}^{h}\right)\right| & =\left|a\left(\mathcal{X}-\mathcal{X}^{h},\left(I-P^{h}\right) \mathcal{X}\right)\right| \\
& \leq\left\|\left(I-F^{*} \Pi\right)\left(\mathcal{X}-\mathcal{X}^{h}\right)\right\|_{X}\left\|\left(I-P^{h}\right) \mathcal{X}\right\|_{X} \\
& \leq 2\left\|\left(I-P^{h}\right) \mathcal{X}\right\|_{X}\left\|\left(I-P^{h}\right) \mathcal{X}\right\|_{X}
\end{aligned}
$$

and the claim follows.

For further derivations we need to define local fields $\sigma\left(\boldsymbol{u}_{k}\right)$ and $\boldsymbol{u}_{k}$ for given $\mathcal{X} \in X$ to satisfy, for each element $K_{k}$ in the mesh,

$$
\begin{aligned}
\sigma\left(\boldsymbol{u}_{k}\right) & =\left(\lambda \nabla \cdot \boldsymbol{u}_{k}\right) I_{d}+\mu\left(\nabla \boldsymbol{u}_{k}+\left(\nabla \boldsymbol{u}_{k}\right)^{T}\right) \quad \text { in } K_{k}, \\
\nabla \cdot \sigma\left(\boldsymbol{u}_{k}\right)+\omega^{2} \boldsymbol{u}_{k} & =\mathbf{0} \quad \text { in } K_{k} \\
-\sigma\left(\boldsymbol{u}_{k}\right) \boldsymbol{n}_{k}-i \eta \boldsymbol{u}_{k} & =\mathcal{X}_{k} \quad \text { on } \partial K_{k},
\end{aligned}
$$


and we define $\sigma\left(\phi_{k}\right)$ and $\phi_{k}$ for given $\mathcal{Y} \in X$ to satisfy, for each $K_{k}$,

$$
\begin{aligned}
\sigma\left(\phi_{k}\right) & =\left(\lambda \nabla \cdot \phi_{k}\right) I_{d}+\mu\left(\nabla \phi_{k}+\left(\nabla \phi_{k}\right)^{T}\right) \quad \text { in } K_{k} \\
\nabla \cdot \sigma\left(\phi_{k}\right)+\omega^{2} \phi_{k} & =\mathbf{0} \quad \text { in } K_{k} \\
-\sigma\left(\phi_{k}\right) \boldsymbol{n}_{k}-i \eta \boldsymbol{\phi}_{k} & =\mathcal{Y}_{k} \quad \text { on } \partial K_{k} .
\end{aligned}
$$

We introduce an auxiliary sesquilinear form $a_{0}: X \times X \rightarrow \mathbb{C}$ given by

$$
a_{0}(\mathcal{X}, \mathcal{Y})=\sum_{k=1}^{N} \int_{\partial K_{k}} i\left(\sigma\left(\bar{\phi}_{k}\right) \boldsymbol{n}_{k} \cdot \hat{\boldsymbol{u}}-\hat{\sigma}(\boldsymbol{u}) \boldsymbol{n}_{k} \cdot \overline{\boldsymbol{\phi}}_{k}\right) \mathrm{d} S
$$

where $\hat{\boldsymbol{u}}$ and $\hat{\sigma}(\boldsymbol{u})$ are the previously defined numerical fluxes.

Now we can establish the following connection between the sesquilinear forms $a_{0}$ and $a$.

Lemma 3.4. The sesquilinear forms $a_{0}($ see (3.13)) and a (see (2.20)) satisfy

$$
a(\mathcal{X}, \mathcal{Y})=a_{0}(\mathcal{X}, \mathcal{Y})+\sum_{k=1}^{N} \int_{\Gamma_{k}} \frac{1}{2} \eta^{-1} F_{k}\left(\mathcal{X}_{k}\right) \cdot \overline{F_{k}\left(\mathcal{Y}_{k}\right)} \mathrm{d} S
$$

for all $\mathcal{X}, \mathcal{Y} \in X$.

Proof. Expanding the sum in (3.13) over interior and exterior faces, and summing over the elements we obtain

$$
\begin{aligned}
a_{0}(\mathcal{X}, \mathcal{Y})= & \sum_{k=1}^{N} \sum_{j=1, j \neq k}^{N} \int_{\sum_{k, j}}\left[\sigma\left(\overline{\boldsymbol{\phi}}_{k}\right) \boldsymbol{n}_{k} \cdot \hat{\boldsymbol{u}}_{k}-\hat{\sigma}\left(\boldsymbol{u}_{k}\right) \boldsymbol{n}_{k} \cdot \overline{\boldsymbol{\phi}}_{k}\right] \mathrm{d} S \\
& +\sum_{k=1}^{N} \int_{\Gamma_{k}}\left[\sigma\left(\overline{\boldsymbol{\phi}}_{k}\right) \boldsymbol{n}_{k} \cdot \hat{\boldsymbol{u}}_{k}-\hat{\sigma}\left(\boldsymbol{u}_{k}\right) \boldsymbol{n}_{k} \cdot \overline{\boldsymbol{\phi}}_{k}\right] \mathrm{d} S \\
= & \sum_{k=1}^{N} \sum_{j=1, j \neq k}^{N} \frac{1}{2}\left[\int_{\sum_{k, j}} \eta^{-1} \mathcal{X}_{k} \cdot \overline{\mathcal{Y}}_{k} \mathrm{~d} S-\int_{\sum_{k, j}} \eta^{-1} \mathcal{X}_{j} \cdot \overline{F_{k}\left(\mathcal{Y}_{k}\right)} \mathrm{d} S\right] \\
& +\sum_{k=1}^{N} \frac{1}{2}\left[\int_{\Gamma_{k}} \eta^{-1} \mathcal{X}_{k} \cdot \overline{\mathcal{Y}}_{k} \mathrm{~d} S-\int_{\Gamma_{k}} \eta^{-1} F_{k}\left(\mathcal{X}_{k}\right) \cdot \overline{F_{k}\left(\mathcal{Y}_{k}\right)} \mathrm{d} S\right] \\
= & a(\mathcal{X}, \mathcal{Y})-\sum_{k=1}^{N} \int_{\Gamma_{k}} \frac{1}{2} \eta^{-1} F_{k}\left(\mathcal{X}_{k}\right) \cdot \overline{F_{k}\left(\mathcal{Y}_{k}\right)} \mathrm{d} S .
\end{aligned}
$$

Lemma 3.5. The sesquilinear form (3.13) can be written as

$a_{0}(\mathcal{X}, \mathcal{Y})=\sum_{f: \text { interior }} \int_{f} i\left(\llbracket \sigma\left(\overline{\boldsymbol{\phi}}_{f}\right) \rrbracket \cdot \hat{\boldsymbol{u}}_{f}-\hat{\sigma}\left(\boldsymbol{u}_{f}\right) \boldsymbol{n}_{f} \cdot \llbracket \overline{\boldsymbol{\phi}}_{f} \rrbracket \boldsymbol{n}_{f}\right) \mathrm{d} S+\sum_{f: \text { exterior }} \int_{f} i\left(\sigma\left(\overline{\boldsymbol{\phi}}_{f}\right) \boldsymbol{n}_{f} \cdot \boldsymbol{u}_{f}-\sigma\left(\boldsymbol{u}_{f}\right) \boldsymbol{n}_{f} \cdot \overline{\boldsymbol{\phi}}_{f}\right) \mathrm{d} S$.

Proof. Equation (3.13) can be separated into two parts (interior and boundary faces) as follows

$$
a_{0}(\mathcal{X}, \mathcal{Y})=\sum_{k=1}^{N} \sum_{j=1, j \neq k}^{N} \int_{\Sigma_{k, j}} i\left(\sigma\left(\overline{\boldsymbol{\phi}}_{k}\right) \boldsymbol{n}_{k} \cdot \hat{\boldsymbol{u}}-\hat{\sigma}(\boldsymbol{u}) \boldsymbol{n}_{k} \cdot \overline{\boldsymbol{\phi}}_{k}\right) \mathrm{d} S+\sum_{k=1}^{N} \int_{\Gamma_{k}} i\left(\sigma\left(\overline{\boldsymbol{\phi}}_{k}\right) \boldsymbol{n}_{k} \cdot \boldsymbol{u}_{k}-\sigma\left(\boldsymbol{u}_{k}\right) \boldsymbol{n}_{k} \cdot \overline{\boldsymbol{\phi}}_{k}\right) \mathrm{d} S .
$$


Let us investigate two neighboring elements $K_{k}$ and $K_{j}$ with a common face $\Sigma_{k, j}$ and normal $\boldsymbol{n}_{k}$. The contribution to (3.15) for this face is from $\Sigma_{k, j}$ and $\Sigma_{j, k}$ so the first terms in the right hand side of (3.15) can be written as

$$
\int_{\Sigma_{k, j}} i \sigma\left(\overline{\boldsymbol{\phi}}_{k}\right) \boldsymbol{n}_{k} \cdot \hat{\boldsymbol{u}} \mathrm{d} S+\int_{\Sigma_{j, k}} i \sigma\left(\overline{\boldsymbol{\phi}}_{j}\right) \boldsymbol{n}_{j} \cdot \hat{\boldsymbol{u}} \mathrm{d} S=\int_{f: \text { interior }} i\left(\llbracket \sigma\left(\overline{\boldsymbol{\phi}}_{f}\right) \rrbracket\right) \cdot \hat{\boldsymbol{u}}_{f} \mathrm{~d} S,
$$

where $f$ denotes the face $\Sigma_{k, j}$ and $\hat{\boldsymbol{u}}_{f}$ is the numerical flux on that face. The second term in the right hand side (inside the first integral) of (3.15) can be written (now without the integral for the sake of compactness), as

$$
\begin{aligned}
-\hat{\sigma}(\boldsymbol{u}) \boldsymbol{n}_{k} \cdot \overline{\boldsymbol{\phi}}_{k}-\hat{\sigma}(\boldsymbol{u}) \boldsymbol{n}_{j} \cdot \overline{\boldsymbol{\phi}}_{j} & =-\hat{\sigma}(\boldsymbol{u}) \boldsymbol{n}_{k} \cdot\left(\bar{\phi}_{k} \otimes \boldsymbol{n}_{k}\right) \boldsymbol{n}_{k}-\hat{\sigma}(\boldsymbol{u}) \boldsymbol{n}_{j} \cdot\left(\bar{\phi}_{j} \otimes \boldsymbol{n}_{j}\right) \boldsymbol{n}_{j} \\
& =-\hat{\sigma}(\boldsymbol{u}) \boldsymbol{n}_{k} \cdot\left(\left(\bar{\phi}_{k} \otimes \boldsymbol{n}_{k}\right) \boldsymbol{n}_{k}-\left(\bar{\phi}_{j} \otimes \boldsymbol{n}_{j}\right) \boldsymbol{n}_{j}\right) \\
& =-\hat{\sigma}(\boldsymbol{u}) \boldsymbol{n}_{k} \cdot\left(\left(\bar{\phi}_{k} \otimes \boldsymbol{n}_{k}\right)+\left(\bar{\phi}_{j} \otimes \boldsymbol{n}_{j}\right)\right) \boldsymbol{n}_{k} \\
& =-\hat{\sigma}(\boldsymbol{u}) \boldsymbol{n}_{k} \cdot \llbracket \overline{\boldsymbol{\phi}}_{k} \rrbracket \boldsymbol{n}_{k} .
\end{aligned}
$$

Then the first integral in (3.15) can be written in terms of the interior faces as

$$
\sum_{k=1}^{N} \sum_{j=1, j \neq k}^{N} \int_{\Sigma_{k, j}} i\left(\sigma\left(\overline{\boldsymbol{\phi}}_{k}\right) \boldsymbol{n}_{k} \cdot \hat{\boldsymbol{u}}-\hat{\sigma}(\boldsymbol{u}) \boldsymbol{n}_{k} \cdot \overline{\boldsymbol{\phi}}_{k}\right) \mathrm{d} S=\sum_{f: \text { interior }} \int_{f} i\left(\llbracket \sigma\left(\overline{\boldsymbol{\phi}}_{f}\right) \rrbracket \cdot \hat{\boldsymbol{u}}_{f}-\hat{\sigma}\left(\boldsymbol{u}_{f}\right) \boldsymbol{n} \cdot \llbracket \overline{\boldsymbol{\phi}}_{f} \rrbracket \boldsymbol{n}\right) \mathrm{d} S
$$

where $f$ emphasizes association to a face. Now using (3.18) in (3.15) the claim follows.

Theorem 3.6. The real part of $a(\mathcal{X}, \mathcal{X})$ can be written as

$$
\begin{aligned}
\Re\{a(\mathcal{X}, \mathcal{X})\}= & \sum_{f: \text { interior }} \int_{f} \frac{1}{2}\left(\llbracket \sigma\left(\overline{\boldsymbol{u}}_{f}\right) \rrbracket \cdot \eta^{-1} \llbracket \sigma\left(\boldsymbol{u}_{f}\right) \rrbracket+\eta \llbracket \boldsymbol{u}_{f} \rrbracket \boldsymbol{n}_{f} \cdot \llbracket \overline{\boldsymbol{u}}_{f} \rrbracket \boldsymbol{n}_{f}\right) \mathrm{d} S \\
& +\sum_{k=1}^{N} \int_{\Gamma_{k}} \frac{1}{4}\left(\eta^{-1} \mathcal{X}_{k}\right) \cdot \overline{\mathcal{X}}_{k} \mathrm{~d} S+\sum_{k=1}^{N} \int_{\Gamma_{k}} \frac{1}{4}\left(\eta^{-1} F_{k}\left(\mathcal{X}_{k}\right)\right) \cdot \overline{F_{k}\left(\mathcal{X}_{k}\right)} \mathrm{d} S .
\end{aligned}
$$

Proof. Due to Lemma 3.4 we can write

$$
a(\mathcal{X}, \mathcal{X})=a_{0}(\mathcal{X}, \mathcal{X})+\sum_{k=1}^{N} \int_{\Gamma_{k}} \frac{1}{2}\left(\eta^{-1} F_{k}\left(\mathcal{X}_{k}\right)\right) \cdot \overline{F_{k}\left(\mathcal{X}_{k}\right)} \mathrm{d} S
$$

Next we take $\sigma\left(\phi_{k}\right)=\sigma\left(\boldsymbol{u}_{k}\right)$ and $\boldsymbol{\phi}_{k}=\boldsymbol{u}_{k}$ in (3.14). Hence, we obtain

$$
a_{0}(\mathcal{X}, \mathcal{X})=\sum_{f: \text { interior }} \int_{f} i\left(\llbracket \sigma\left(\overline{\boldsymbol{u}}_{f}\right) \rrbracket \cdot \hat{\boldsymbol{u}}_{f}-\hat{\sigma}\left(\boldsymbol{u}_{f}\right) \boldsymbol{n}_{f} \cdot \llbracket \overline{\boldsymbol{u}}_{f} \rrbracket \boldsymbol{n}_{f}\right) \mathrm{d} S+\sum_{f: \text { exterior }} \int_{f} i\left(\sigma\left(\overline{\boldsymbol{u}}_{f}\right) \boldsymbol{n}_{f} \cdot \boldsymbol{u}_{f}-\sigma\left(\boldsymbol{u}_{f}\right) \boldsymbol{n}_{f} \cdot \overline{\boldsymbol{u}}_{f}\right) \mathrm{d} S .
$$

Using the definitions of averages and jumps on the interior face we have

$$
\begin{aligned}
\llbracket \sigma\left(\overline{\boldsymbol{u}}_{f}\right) \rrbracket \cdot \hat{\boldsymbol{u}}_{f}-\hat{\sigma}\left(\boldsymbol{u}_{f}\right) \boldsymbol{n}_{f} \cdot \llbracket \overline{\boldsymbol{u}}_{f} \rrbracket \boldsymbol{n}_{f}=\llbracket \sigma\left(\overline{\boldsymbol{u}}_{f}\right) \rrbracket \cdot\left\{\left\{\boldsymbol{u}_{f}\right\}\right\}-\frac{i}{2} \llbracket \sigma\left(\overline{\boldsymbol{u}}_{f}\right) \rrbracket \cdot \eta^{-1} \llbracket \sigma\left(\boldsymbol{u}_{f}\right) \rrbracket \\
-\{\{\sigma(\boldsymbol{u})\}\} \boldsymbol{n}_{f} \cdot \llbracket \overline{\boldsymbol{u}}_{f} \rrbracket \boldsymbol{n}_{f}-\frac{i}{2} \eta \llbracket \boldsymbol{u}_{f} \rrbracket \boldsymbol{n}_{f} \cdot \llbracket \overline{\boldsymbol{u}}_{f} \rrbracket \boldsymbol{n}_{f} .
\end{aligned}
$$


Let us assume that there are two elements $K_{k}$ and $K_{j}$ which share a common face. Since we sum over two elements over the interior faces we have

$$
\begin{aligned}
\llbracket \sigma\left(\overline{\boldsymbol{u}}_{f}\right) \rrbracket \cdot\left\{\left\{\boldsymbol{u}_{f}\right\}\right\}-\{\{\sigma(\boldsymbol{u})\}\} \boldsymbol{n}_{f} \cdot \llbracket \overline{\boldsymbol{u}}_{f} \rrbracket \boldsymbol{n}_{f}= & \frac{1}{2}\left(\left(\sigma\left(\overline{\boldsymbol{u}}_{k}\right) \boldsymbol{n}_{k}+\sigma\left(\overline{\boldsymbol{u}}_{j}\right) \boldsymbol{n}_{j}\right) \cdot\left(\boldsymbol{u}_{k}+\boldsymbol{u}_{j}\right)\right. \\
& \left.-\left(\sigma\left(\boldsymbol{u}_{k}\right)+\sigma\left(\boldsymbol{u}_{j}\right)\right) \boldsymbol{n}_{k} \cdot\left(\overline{\boldsymbol{u}}_{k} \otimes \boldsymbol{n}_{k}+\overline{\boldsymbol{u}}_{j} \otimes \boldsymbol{n}_{j}\right) \boldsymbol{n}_{k}\right) \\
= & \frac{1}{2}\left(\left(\sigma\left(\overline{\boldsymbol{u}}_{k}\right) \boldsymbol{n}_{k}+\sigma\left(\overline{\boldsymbol{u}}_{j}\right) \boldsymbol{n}_{j}\right) \cdot\left(\boldsymbol{u}_{k}+\boldsymbol{u}_{j}\right)-\left(\sigma\left(\boldsymbol{u}_{k}\right)+\sigma\left(\boldsymbol{u}_{j}\right)\right) \boldsymbol{n}_{k} \cdot\left(\overline{\boldsymbol{u}}_{k}-\overline{\boldsymbol{u}}_{j}\right)\right) \\
= & \frac{1}{2}\left(\sigma\left(\overline{\boldsymbol{u}}_{k}\right) \boldsymbol{n}_{k} \cdot \boldsymbol{u}_{k}+\sigma\left(\overline{\boldsymbol{u}}_{j}\right) \boldsymbol{n}_{j} \cdot \boldsymbol{u}_{j}+\sigma\left(\overline{\boldsymbol{u}}_{k}\right) \boldsymbol{n}_{k} \cdot \boldsymbol{u}_{j}+\sigma\left(\overline{\boldsymbol{u}}_{j}\right) \boldsymbol{n}_{j} \cdot \boldsymbol{u}_{k}\right. \\
& \left.-\sigma\left(\boldsymbol{u}_{k}\right) \boldsymbol{n}_{k} \cdot \overline{\boldsymbol{u}}_{k}+\sigma\left(\boldsymbol{u}_{j}\right) \boldsymbol{n}_{k} \cdot \overline{\boldsymbol{u}}_{j}+\sigma\left(\boldsymbol{u}_{k}\right) \boldsymbol{n}_{k} \cdot \overline{\boldsymbol{u}}_{j}-\sigma\left(\boldsymbol{u}_{j}\right) \boldsymbol{n}_{k} \cdot \overline{\boldsymbol{u}}_{k}\right) .
\end{aligned}
$$

Note that

$$
\begin{aligned}
\Im\left\{\left(\sigma\left(\overline{\boldsymbol{u}}_{j}\right) \boldsymbol{n}_{j} \cdot \boldsymbol{u}_{k}+\sigma\left(\boldsymbol{u}_{k}\right) \boldsymbol{n}_{k} \cdot \overline{\boldsymbol{u}}_{j}\right)+\left(-\sigma\left(\boldsymbol{u}_{j}\right) \boldsymbol{n}_{k} \cdot \overline{\boldsymbol{u}}_{k}+\sigma\left(\overline{\boldsymbol{u}}_{k}\right) \boldsymbol{n}_{k} \cdot \boldsymbol{u}_{j}\right)\right\}=\Im\left\{\left(\sigma\left(\overline{\boldsymbol{u}}_{j}\right) \boldsymbol{n}_{j} \cdot \boldsymbol{u}_{k}-\sigma\left(\boldsymbol{u}_{k}\right) \boldsymbol{n}_{j} \cdot \overline{\boldsymbol{u}}_{j}\right)\right. \\
\left.+\left(\sigma\left(\boldsymbol{u}_{j}\right) \boldsymbol{n}_{j} \cdot \overline{\boldsymbol{u}}_{k}-\sigma\left(\overline{\boldsymbol{u}}_{k}\right) \boldsymbol{n}_{j} \cdot \boldsymbol{u}_{j}\right)\right\}=0
\end{aligned}
$$

where $\Im\{\cdot\}$ takes the imaginary part. Then equation (3.23) can be written as

$$
\begin{aligned}
\Im\left\{\llbracket \sigma\left(\overline{\boldsymbol{u}}_{f}\right) \rrbracket \cdot\left\{\left\{\boldsymbol{u}_{f}\right\}\right\}-\{\{\sigma(\boldsymbol{u})\}\} \boldsymbol{n}_{f} \cdot \llbracket \overline{\boldsymbol{u}}_{f} \rrbracket \boldsymbol{n}_{f}\right\}= & \frac{1}{2} \Im\left\{\sigma\left(\overline{\boldsymbol{u}}_{k}\right) \boldsymbol{n}_{k} \cdot \boldsymbol{u}_{k}+\sigma\left(\overline{\boldsymbol{u}}_{j}\right) \boldsymbol{n}_{j} \cdot \boldsymbol{u}_{j}\right. \\
& \left.-\sigma\left(\boldsymbol{u}_{k}\right) \boldsymbol{n}_{k} \cdot \overline{\boldsymbol{u}}_{k}+\sigma\left(\boldsymbol{u}_{j}\right) \boldsymbol{n}_{k} \cdot \overline{\boldsymbol{u}}_{j}\right\} \\
= & \frac{1}{2} \Im\left\{\left(\sigma\left(\overline{\boldsymbol{u}}_{k}\right) \boldsymbol{n}_{k} \cdot \boldsymbol{u}_{k}-\sigma\left(\boldsymbol{u}_{k}\right) \boldsymbol{n}_{k} \cdot \overline{\boldsymbol{u}}_{k}\right)\right. \\
& \left.+\left(\sigma\left(\overline{\boldsymbol{u}}_{j}\right) \boldsymbol{n}_{j} \cdot \boldsymbol{u}_{j}+\sigma\left(\boldsymbol{u}_{j}\right) \boldsymbol{n}_{k} \cdot \overline{\boldsymbol{u}}_{j}\right)\right\} \\
= & \frac{1}{2} \Im\left\{\left(\sigma\left(\overline{\boldsymbol{u}}_{k}\right) \boldsymbol{n}_{k} \cdot \boldsymbol{u}_{k}-\sigma\left(\boldsymbol{u}_{k}\right) \boldsymbol{n}_{k} \cdot \overline{\boldsymbol{u}}_{k}\right)\right. \\
& \left.+\left(\sigma\left(\overline{\boldsymbol{u}}_{j}\right) \boldsymbol{n}_{j} \cdot \boldsymbol{u}_{j}-\sigma\left(\boldsymbol{u}_{j}\right) \boldsymbol{n}_{j} \cdot \overline{\boldsymbol{u}}_{j}\right)\right\} .
\end{aligned}
$$

Using equations (3.25) and (3.14) we have

$$
\begin{aligned}
\Re\left\{a_{0}(\mathcal{X}, \mathcal{X})\right\}= & \Re\left\{\sum_{k=1}^{N} \int_{\partial K_{k}} \frac{i}{2}\left(\sigma\left(\overline{\boldsymbol{u}}_{k}\right) \boldsymbol{n}_{k} \cdot \boldsymbol{u}_{k}-\sigma\left(\boldsymbol{u}_{k}\right) \boldsymbol{n}_{k} \cdot \overline{\boldsymbol{u}}_{k}\right) \mathrm{d} S\right\} \\
& +\Re\left\{\sum_{f: \text { exterior }} \int_{f} \frac{i}{2}\left(\sigma\left(\overline{\boldsymbol{u}}_{f}\right) \boldsymbol{n}_{f} \cdot \boldsymbol{u}_{f}-\sigma\left(\boldsymbol{u}_{f}\right) \boldsymbol{n}_{f} \cdot \overline{\boldsymbol{u}}_{f}\right) \mathrm{d} S\right\} \\
& +\sum_{f: \text { interior }} \int_{f}\left(\frac{1}{2} \llbracket \sigma\left(\overline{\boldsymbol{u}}_{f}\right) \rrbracket \cdot \eta^{-1} \llbracket \sigma\left(\boldsymbol{u}_{f}\right) \rrbracket+\frac{1}{2} \eta \llbracket \boldsymbol{u}_{f} \rrbracket \boldsymbol{n}_{f} \cdot \llbracket \overline{\boldsymbol{u}}_{f} \rrbracket \boldsymbol{n}_{f}\right) \mathrm{d} S .
\end{aligned}
$$

By Betti's third identity the first integral term vanishes and we have

$$
\begin{aligned}
\Re\left\{a_{0}(\mathcal{X}, \mathcal{X})\right\}= & \sum_{f: \text { exterior }} \int_{f} \frac{i}{2}\left(\sigma\left(\overline{\boldsymbol{u}}_{f}\right) \boldsymbol{n}_{f} \cdot \boldsymbol{u}_{f}-\sigma\left(\boldsymbol{u}_{f}\right) \boldsymbol{n}_{f} \cdot \overline{\boldsymbol{u}}_{f}\right) \mathrm{d} S \\
& +\sum_{f: \text { interior }} \int_{f} \frac{1}{2}\left(\llbracket \sigma\left(\overline{\boldsymbol{u}}_{f}\right) \rrbracket \cdot \eta^{-1} \llbracket \sigma\left(\boldsymbol{u}_{f}\right) \rrbracket+\eta \llbracket \boldsymbol{u}_{f} \rrbracket \boldsymbol{n}_{f} \cdot \llbracket \overline{\boldsymbol{u}}_{f} \rrbracket \boldsymbol{n}_{f}\right) \mathrm{d} S .
\end{aligned}
$$


Using (2.16) in (3.27) we obtain

$$
\begin{aligned}
\Re\left\{a_{0}(\mathcal{X}, \mathcal{X})\right\}= & \int_{\Gamma_{k}} \frac{1}{4} \eta^{-1} \mathcal{X}_{k} \cdot \overline{\mathcal{X}}_{k} \mathrm{~d} S-\int_{\Gamma_{k}} \frac{1}{4} \eta^{-1} F_{k}\left(\mathcal{X}_{k}\right) \cdot \overline{F_{k}\left(\mathcal{X}_{k}\right)} \mathrm{d} S \\
& +\sum_{f: \text { interior }} \int_{f} \frac{1}{2}\left(\llbracket \sigma\left(\overline{\boldsymbol{u}}_{f}\right) \rrbracket \cdot \eta^{-1} \llbracket \sigma\left(\boldsymbol{u}_{f}\right) \rrbracket+\eta \llbracket \boldsymbol{u}_{f} \rrbracket \boldsymbol{n}_{f} \cdot \llbracket \overline{\boldsymbol{u}}_{f} \rrbracket \boldsymbol{n}_{f}\right) \mathrm{d} S .
\end{aligned}
$$

The claim follows when equation (3.28) is plugged into equation (3.20).

Now we can write a global error estimate. To do this we need to define a global approximation to $\boldsymbol{u}$ denoted $\boldsymbol{u}_{h}$. This is defined element by element by requiring that on each element $K_{k}$, the discrete function $\left.\boldsymbol{u}_{h}\right|_{K_{k}}$ satisfies (3.7)-(3.9) with $\mathcal{X}_{k}^{h}$ replacing $\mathcal{X}_{k}$. In the particular case covered by this paper, the wave numbers $\kappa_{P}$ and $\kappa_{S}$ are real, and so

$$
\left.\boldsymbol{u}_{h}\right|_{K_{k}}=\sum_{\ell=1}^{p^{P}} \mathcal{X}_{k, \ell}^{P} \varphi_{k, \ell}^{P}+\sum_{\ell=1}^{p^{S}}\left(\mathcal{X}_{k, \ell}^{S H} \varphi_{k, \ell}^{S H}+\mathcal{X}_{k, \ell}^{S V} \varphi_{k, \ell}^{S V}\right)
$$

where the coefficients are from (2.25) and the basis functions are given in (2.22)-(2.24).

Theorem 3.7. Let $\boldsymbol{u}_{h}$ denote the discrete solution defined (3.29) and let $\boldsymbol{u}$ be the solution of (2.1)-(2.2). Then the following error estimate holds

$$
\begin{aligned}
\sum_{f: \text { interior }} \int_{f} \frac{1}{2}\left(\llbracket \sigma\left(\overline{\boldsymbol{u}}_{f}-\overline{\boldsymbol{u}}_{h, f}\right) \rrbracket \cdot \eta^{-1} \llbracket \sigma\left(\boldsymbol{u}_{f}-\boldsymbol{u}_{h, f}\right) \rrbracket+\eta \llbracket \boldsymbol{u}_{f}-\boldsymbol{u}_{h, f} \rrbracket \boldsymbol{n}_{f} \cdot \llbracket \overline{\boldsymbol{u}}_{f}-\overline{\boldsymbol{u}}_{h, f} \rrbracket \boldsymbol{n}_{f}\right) \mathrm{d} S \\
\quad+\sum_{k=1}^{N} \int_{\Gamma_{k}} \frac{1}{4}\left|\eta^{-\frac{1}{2}} F_{k}\left(\mathcal{X}_{k}-\mathcal{X}_{k}^{h}\right)\right|^{2} \mathrm{~d} A+\sum_{k=1}^{N} \int_{\Gamma_{k}} \frac{1}{4}\left|\eta^{-\frac{1}{2}}\left(\mathcal{X}_{k}-\mathcal{X}_{k}^{h}\right)\right|^{2} \mathrm{~d} S \\
\leq 2\left\|\left(I-P^{h}\right) \mathcal{X}\right\|_{X}^{2} .
\end{aligned}
$$

Remark 3.8. This result proves convergence on $\Gamma$ (cf. Cessenat and Després [6]) and convergence in a DG norm at the same time. Note that it holds for a general domain and a general regular mesh. In the next section we shall prove convergence in a standard norm, but on a restricted class of elements and domains.

Remark 3.9. The conclusion of this theorem is better than the corresponding estimate in Lemma 3.4. of [4] because it includes an estimate for the $\mathrm{L}_{\eta}^{2}(\Gamma)$ norm of $\mathcal{X}_{k}-\mathcal{X}_{k}^{h}$ on the boundary. Unfortunately in the proof of Lemma 3.4. of [4], a factor $1 / 2$ is missing in the step where averages are expanded and this changes the constants in the estimate as well as removing the boundary estimate term. Using the correct expansion shows that (3.13) of [4] holds with the factor (1/2) multiplying the boundary flux terms replaced by (1/4). In addition, a term in $\int_{\Gamma_{k}} \frac{1}{4}\left|\eta^{-\frac{1}{2}}\left(\mathcal{X}_{k}-\mathcal{X}_{k}^{h}\right)\right|^{2} \mathrm{~d} S$ should also be added. From then on the conclusions of [4] hold with appropriate changes to the constants.

Proof. Due to the Theorem 3.6 we have

$$
\begin{aligned}
\sum_{f: \text { interior }} \int_{f} & \frac{1}{2}\left(\llbracket \sigma\left(\overline{\boldsymbol{u}}_{f}-\overline{\boldsymbol{u}}_{h, f}\right) \rrbracket \cdot \eta^{-1} \llbracket \sigma\left(\boldsymbol{u}_{f}-\boldsymbol{u}_{h, f}\right) \rrbracket+\eta \llbracket \boldsymbol{u}_{f}-\boldsymbol{u}_{h, f} \rrbracket \boldsymbol{n}_{f} \cdot \llbracket \overline{\boldsymbol{u}}_{f}-\overline{\boldsymbol{u}}_{h, f} \rrbracket \boldsymbol{n}_{f}\right) \mathrm{d} S \\
& +\sum_{k=1}^{N} \int_{\Gamma_{k}} \frac{1}{4}\left(\eta^{-1}\left(\mathcal{X}_{k}-\mathcal{X}_{k}^{h}\right)\right) \cdot\left(\overline{\mathcal{X}}_{k}-\overline{\mathcal{X}}_{k}^{h}\right) \mathrm{d} S+\sum_{k=1}^{N} \int_{\Gamma_{k}} \frac{1}{4}\left(\eta^{-1} F_{k}\left(\mathcal{X}_{k}-\mathcal{X}_{k}^{h}\right)\right) \cdot \overline{F_{k}\left(\mathcal{X}_{k}-\mathcal{X}_{k}^{h}\right)} \mathrm{d} S \\
= & \Re\left\{a\left(\mathcal{X}-\mathcal{X}^{h}, \mathcal{X}-\mathcal{X}^{h}\right)\right\} \leq\left|a\left(\mathcal{X}-\mathcal{X}^{h}, \mathcal{X}-\mathcal{X}^{h}\right)\right| .
\end{aligned}
$$


Furthermore, by Theorem 3.3 we obtain

$$
\left|a\left(\mathcal{X}-\mathcal{X}^{h}, \mathcal{X}-\mathcal{X}^{h}\right)\right| \leq 2\left\|\left(I-P^{h}\right) \mathcal{X}\right\|_{X}^{2} .
$$

Lemmas 3.2 and 3.3 of [6] are now a corollary of Theorem 3.7:

Corollary 3.10 (Lems. 3.2, 3.3 in [6]). The following estimate holds on $\Gamma$ :

$$
\left\|\mathcal{X}-\mathcal{X}^{h}\right\|_{L_{\eta}^{2}(\Gamma)} \leq 2\left\|\left(I-P^{h}\right) \mathcal{X}\right\|_{X}
$$

where

$$
\left\|\mathcal{X}-\mathcal{X}^{h}\right\|_{\mathrm{L}_{\eta}^{2}(\Gamma)}^{2}=\sum_{k=1}^{N} \int_{\Gamma_{k}} \frac{1}{2}\left(\eta^{-1}\left(\mathcal{X}-\mathcal{X}^{h}\right)\right) \cdot \overline{\left(\mathcal{X}-\mathcal{X}^{h}\right)} \mathrm{d} S .
$$

\subsection{Error estimate in $\mathrm{L}^{2}(\Omega)$}

In this section we prove an error estimate in $\mathrm{L}^{2}(\Omega)$ for the UWVF for the Navier problem. We start with fundamental lemmas needed when proving the main theorem. We adopt the approach from $[4,7,27]$. We shall denote the norm on $\mathrm{H}^{k}(\Omega)$ by $\|\cdot\|_{k, \Omega}$, and the $\mathrm{L}^{2}(\Omega)$ norm is denoted by $\|\cdot\|_{0, \Omega}$. From now on we assume $\Omega$ be convex polyhedron and the mesh is quasi-uniform so that for each mesh size $h$ we have the bound $h_{K_{k}}^{-1} \leq h^{-1}$ for some $C$ independent of $h$ and $K_{k}$. In addition, we shall assume that there is a constant $C$ independent of $h$ and $\omega$ such that $\|\eta\|_{\infty}<C \omega$ and $\left\|\eta^{-1}\right\|_{\infty}<C \omega^{-1}$ (other choices including $h$ dependent matrices are possible and could be included in the theory at the cost of more complicated notation (see $[13,14]$ for acoustic case)).

We start recalling the definition of the matrix inner product $A: B$, see, for example, [7]. Let $A$ and $B$ be $M \times M$ matrices with entries $a_{k l}$ and $b_{k l}$, respectively, where $l=1, \ldots, M$ and $k=1, \ldots, M$. Then the matrix inner product $A: B$ is

$$
A: B=\sum_{k=1}^{M} \sum_{l=1}^{M} a_{k l} b_{k l} .
$$

We now can write the similar result of Lemma 3(a) in [7], see page 144.

Lemma 3.11 (Lem. 3(a) in [7]). For any $\boldsymbol{u}, \boldsymbol{v} \in \mathrm{H}^{2}(\Omega, \mathcal{C})$ the following relation hold

$$
\sigma(\boldsymbol{u}): \nabla \boldsymbol{v}=\lambda \nabla \cdot \boldsymbol{u} \nabla \cdot \boldsymbol{v}+2 \mu \varepsilon(\boldsymbol{u}): \varepsilon(\boldsymbol{v}) .
$$

Proof. Direct calculation verifies the identity.

Clearly, based on the fact that the matrix inner product produces a scalar (and the operator : is commutative), we notice that

$$
\sigma(\boldsymbol{v}): \nabla \boldsymbol{u}=\lambda \nabla \cdot \boldsymbol{u} \nabla \cdot \boldsymbol{v}+2 \mu \varepsilon(\boldsymbol{u}): \varepsilon(\boldsymbol{v})=\nabla \boldsymbol{v}: \sigma(\boldsymbol{u}) .
$$

We now consider the inhomogeneous Navier equation with the following boundary conditions (dual problem, see $[27])$

$$
\begin{aligned}
& \nabla \cdot \sigma(\boldsymbol{v})+\omega^{2} \rho \boldsymbol{v}=\boldsymbol{\psi} \quad \text { in } \Omega, \\
& \sigma(\boldsymbol{v}) \boldsymbol{n}-i \eta \boldsymbol{v}=\mathbf{0} \quad \text { on } \Gamma \text {, }
\end{aligned}
$$

where $\boldsymbol{\psi}$ is an arbitrary function in $\mathrm{L}^{2}(\Omega)$. Let us recall the regularity estimates for the elastic problem proved in [7]. 
Theorem 3.12 (Thm. 2 in Ref. [7]). Let $\Omega$ be a convex polyhedron or smooth domain. Then the following regularity estimates for $\boldsymbol{v}$ hold:

$$
\begin{aligned}
& \|\boldsymbol{v}\|_{1, \Omega} \leq C\left(1+\omega+\frac{1}{\omega^{2}}\right)\|\boldsymbol{\psi}\|_{0, \Omega}, \\
& \|\boldsymbol{v}\|_{2, \Omega} \leq C\left(1+\omega^{2}+\frac{1}{\omega^{2}}\right)\|\boldsymbol{\psi}\|_{0, \Omega} .
\end{aligned}
$$

Now we can prove the following error estimate using duality.

Theorem 3.13. Let $\boldsymbol{w} \in \mathrm{H}^{2}\left(K_{k}\right), k=1, \ldots, N$, be a solution of the Navier problem on each element. In addition, let $\Omega$ be a convex polyhedron. Then, if $\Omega$ is covered by a regular and quasi-uniform mesh,

$$
\|\boldsymbol{w}\|_{0, \Omega} \leq C h^{-1 / 2}\left(\omega^{\frac{3}{2}}+\omega^{-\frac{5}{2}}\right)\left[\sum_{f \in \Sigma}\left(\left\|\eta^{\frac{1}{2}} \llbracket \boldsymbol{w} \rrbracket \boldsymbol{n}\right\|_{0, f}^{2}+\left\|\eta^{-\frac{1}{2}} \llbracket \sigma(\boldsymbol{w}) \rrbracket\right\|_{0, f}^{2}\right)+\sum_{f \in \Gamma}\left(\left\|\eta^{-\frac{1}{2}} \sigma(\boldsymbol{w}) \boldsymbol{n}+i \eta^{-\frac{1}{2}} \eta \boldsymbol{w}\right\|_{0, f}^{2}\right)\right]^{1 / 2}
$$

where $\|\cdot\|_{0, f}$ is the $\mathrm{L}^{2}$-norm on the face $f$ and $C$ is independent of $\omega, \eta, h$ and $\boldsymbol{w}$.

Proof. Let $\boldsymbol{v}$ satisfy the dual problem shown in (3.35) and (3.36). We take complex conjugate of both sides of equation (3.35) and multiply it by $\boldsymbol{w}$ that satisfies the Navier equation i.e. $\nabla \cdot \sigma(\boldsymbol{w})+\omega^{2} \rho \boldsymbol{w}=0$ in $\Omega$. Then

$$
\left(\nabla \cdot \sigma(\overline{\boldsymbol{v}})+\omega^{2} \rho \overline{\boldsymbol{v}}\right) \cdot \boldsymbol{w}=\overline{\boldsymbol{\psi}} \cdot \boldsymbol{w} .
$$

Integrating both sides element by element, summing over elements and using integration by parts we obtain

$$
(\boldsymbol{w}, \boldsymbol{\psi})=\sum_{k=1}^{N} \int_{K_{k}} \omega^{2} \rho \overline{\boldsymbol{v}} \cdot \boldsymbol{w} \mathrm{d} V+\sum_{k=1}^{N} \int_{\partial K_{k}} \sigma(\overline{\boldsymbol{v}}) \boldsymbol{n} \cdot \boldsymbol{w} \mathrm{d} S-\sum_{k=1}^{N} \int_{K_{k}} \sigma(\overline{\boldsymbol{v}}): \nabla \boldsymbol{w} \mathrm{d} V,
$$

where on the left hand side $(\cdot, \cdot)$ is the $\mathrm{L}^{2}(\Omega)$ inner product. Next we use the relation $\sigma(\overline{\boldsymbol{v}}): \nabla \boldsymbol{w}=\nabla \overline{\boldsymbol{v}}: \sigma(\boldsymbol{w})$ shown in (3.34) to obtain

$$
(\boldsymbol{w}, \boldsymbol{\psi})=\sum_{k=1}^{N} \int_{K_{k}} \omega^{2} \rho \overline{\boldsymbol{v}} \cdot \boldsymbol{w} \mathrm{d} V+\sum_{k=1}^{N} \int_{\partial K_{k}} \sigma(\overline{\boldsymbol{v}}) \boldsymbol{n} \cdot \boldsymbol{w} \mathrm{d} S-\sum_{k=1}^{N} \int_{K_{k}} \nabla \overline{\boldsymbol{v}}: \sigma(\boldsymbol{w}) \mathrm{d} V .
$$

Applying integration by parts to the last term again we have

$$
\begin{aligned}
(\boldsymbol{w}, \boldsymbol{\psi})= & \sum_{k=1}^{N} \int_{K_{k}} \omega^{2} \rho \overline{\boldsymbol{v}} \cdot \boldsymbol{w} \mathrm{d} V+\sum_{k=1}^{N} \int_{\partial K_{k}} \sigma(\overline{\boldsymbol{v}}) \boldsymbol{n} \cdot \boldsymbol{w} \mathrm{d} S \\
& -\sum_{k=1}^{N} \int_{\partial K_{k}} \sigma(\boldsymbol{w}) \boldsymbol{n} \cdot \overline{\boldsymbol{v}} \mathrm{d} S+\sum_{k=1}^{N} \int_{K_{k}} \overline{\boldsymbol{v}} \cdot \nabla \cdot \sigma(\boldsymbol{w}) \mathrm{d} V \\
= & \sum_{k=1}^{N} \int_{K_{k}}\left(\omega^{2} \rho \boldsymbol{w}+\nabla \cdot \sigma(\boldsymbol{w})\right) \cdot \overline{\boldsymbol{v}} \mathrm{d} V \\
& +\sum_{k=1}^{N} \int_{\partial K_{k}} \sigma(\overline{\boldsymbol{v}}) \boldsymbol{n} \cdot \boldsymbol{w} \mathrm{d} S-\sum_{k=1}^{N} \int_{\partial K_{k}} \sigma(\boldsymbol{w}) \boldsymbol{n} \cdot \overline{\boldsymbol{v}} \mathrm{d} S .
\end{aligned}
$$


Because $\boldsymbol{w}$ satisfies the homogeneous Navier equation (i.e. $\omega^{2} \rho \boldsymbol{w}+\nabla \cdot \sigma(\boldsymbol{w})=0$ ) we can write

$$
\begin{aligned}
(\boldsymbol{w}, \boldsymbol{\psi}) & =\sum_{k=1}^{N} \int_{\partial K_{k}} \sigma(\overline{\boldsymbol{v}}) \boldsymbol{n} \cdot \boldsymbol{w} \mathrm{d} S-\sum_{k=1}^{N} \int_{\partial K_{k}} \sigma(\boldsymbol{w}) \boldsymbol{n} \cdot \overline{\boldsymbol{v}} \mathrm{d} S \\
& =: \sum_{k=1}^{N}\langle\boldsymbol{w}, \sigma(\boldsymbol{v}) \boldsymbol{n}\rangle_{\partial K_{k}}-\sum_{k=1}^{N}\langle\sigma(\boldsymbol{w}) \boldsymbol{n}, \boldsymbol{v}\rangle_{\partial K_{k}}
\end{aligned}
$$

where

$$
\langle\boldsymbol{w}, \sigma(\boldsymbol{v}) \boldsymbol{n}\rangle_{\partial K_{k}}=\int_{\partial K_{k}} \sigma(\overline{\boldsymbol{v}}) \boldsymbol{n} \cdot \boldsymbol{w} \mathrm{d} S .
$$

Summing over the element interior faces denoted by $\Sigma$ and exterior boundary $\Gamma$, recalling that $\llbracket \sigma(\boldsymbol{w}) \rrbracket$ is a vector while $\llbracket \boldsymbol{w} \rrbracket$ is a matrix, we obtain

$$
(\boldsymbol{w}, \boldsymbol{\psi})=\sum_{f \in \Sigma}\left(\langle\llbracket \boldsymbol{w} \rrbracket \boldsymbol{n}, \sigma(\boldsymbol{v}) \boldsymbol{n}\rangle_{f}-\langle\llbracket \sigma(\boldsymbol{w}) \rrbracket, \boldsymbol{v}\rangle_{f}\right)+\sum_{f \in \Gamma}\left(\langle\boldsymbol{w}, \sigma(\boldsymbol{v}) \boldsymbol{n}\rangle_{f}-\langle\sigma(\boldsymbol{w}) \boldsymbol{n}, \boldsymbol{v}\rangle_{f}\right),
$$

where $\llbracket \cdot \rrbracket$ is the jump as given in equation (2.8). Taking into account the boundary condition (3.36) we obtain

$$
(\boldsymbol{w}, \boldsymbol{\psi})=\sum_{f \in \Sigma}\left(\langle\llbracket \boldsymbol{w} \rrbracket \boldsymbol{n}, \sigma(\boldsymbol{v}) \boldsymbol{n}\rangle_{f}-\langle\llbracket \sigma(\boldsymbol{w}) \rrbracket, \boldsymbol{v}\rangle_{f}\right)+\sum_{f \in \Gamma}\left(\langle\boldsymbol{w}, i \eta \boldsymbol{v}\rangle_{f}-\langle\sigma(\boldsymbol{w}) \boldsymbol{n}, \boldsymbol{v}\rangle_{f}\right) .
$$

Rearranging terms and using the fact that $\langle x, i y\rangle=\langle-i x, y\rangle$ we obtain

$$
\begin{aligned}
& (\boldsymbol{w}, \boldsymbol{\psi})=\sum_{f \in \Sigma}\left(\left\langle\eta^{\frac{1}{2}} \llbracket \boldsymbol{w} \rrbracket \boldsymbol{n}, \eta^{-\frac{1}{2}} \sigma(\boldsymbol{v}) \boldsymbol{n}\right\rangle_{f}-\left\langle\eta^{-\frac{1}{2}} \llbracket \sigma(\boldsymbol{w}) \rrbracket, \eta^{\frac{1}{2}} \boldsymbol{v}\right\rangle_{f}\right)+\sum_{f \in \Gamma}\left(\left\langle-i \eta^{-\frac{1}{2}} \eta \boldsymbol{w}, \eta^{\frac{1}{2}} \boldsymbol{v}\right\rangle_{f}-\left\langle\eta^{-\frac{1}{2}} \sigma(\boldsymbol{w}) \boldsymbol{n}, \eta^{\frac{1}{2}} \boldsymbol{v}\right\rangle_{f}\right) \\
& =\sum_{f \in \Sigma}\left(\left\langle\eta^{\frac{1}{2}} \llbracket \boldsymbol{w} \rrbracket \boldsymbol{n}, \eta^{-\frac{1}{2}} \sigma(\boldsymbol{v}) \boldsymbol{n}\right\rangle_{f}-\left\langle\eta^{-\frac{1}{2}} \llbracket \sigma(\boldsymbol{w}) \rrbracket, \eta^{\frac{1}{2}} \boldsymbol{v}\right\rangle_{f}\right)-\sum_{f \in \Gamma}\left(\left\langle i \eta^{-\frac{1}{2}} \eta \boldsymbol{w}+\eta^{-\frac{1}{2}} \sigma(\boldsymbol{w}) \boldsymbol{n}, \eta^{\frac{1}{2}} \boldsymbol{v}\right\rangle_{f}\right) .
\end{aligned}
$$

Using the Cauchy-Schwarz inequality we have

$|(\boldsymbol{w}, \boldsymbol{\psi})| \leq \sum_{f \in \Sigma}\left\|\eta^{\frac{1}{2}} \llbracket \boldsymbol{w} \rrbracket \boldsymbol{n}\right\|_{0, f}\left\|\eta^{-\frac{1}{2}} \sigma(\boldsymbol{v}) \boldsymbol{n}\right\|_{0, f}+\left\|\eta^{-\frac{1}{2}} \llbracket \sigma(\boldsymbol{w}) \rrbracket\right\|_{0, f}\left\|\eta^{\frac{1}{2}} \boldsymbol{v}\right\|_{0, f}+\sum_{f \in \Gamma}\left\|\eta^{-\frac{1}{2}} \sigma(\boldsymbol{w}) \boldsymbol{n}+i \eta^{-\frac{1}{2}} \eta \boldsymbol{w}\right\|_{0, f}\left\|\eta^{\frac{1}{2}} \boldsymbol{v}\right\|_{0, f}$

Applying the Cauchy-Schwarz inequality again we obtain

$$
\begin{aligned}
|(\boldsymbol{w}, \boldsymbol{\psi})| \leq & {\left[\sum_{f \in \Sigma}\left(\left\|\eta^{\frac{1}{2}} \llbracket \boldsymbol{w} \rrbracket \boldsymbol{n}\right\|_{0, f}^{2}+\left\|\eta^{-\frac{1}{2}} \llbracket \sigma(\boldsymbol{w}) \rrbracket\right\|_{0, f}^{2}\right)+\sum_{f \in \Gamma}\left(\left\|\eta^{-\frac{1}{2}} \sigma(\boldsymbol{w}) \boldsymbol{n}+i \eta^{-\frac{1}{2}} \eta \boldsymbol{w}\right\|_{0, f}^{2}\right)\right]^{1 / 2} } \\
& \times\left[\sum_{f}\left(\left\|\eta^{\frac{1}{2}} \boldsymbol{v}\right\|_{0, f}^{2}+\left\|\eta^{-\frac{1}{2}} \sigma(\boldsymbol{v}) \boldsymbol{n}\right\|_{0, f}^{2}\right)\right]^{1 / 2} \cdot
\end{aligned}
$$

Furthermore, we define \|\| $\boldsymbol{v} \| \mid$ by

$$
\|\boldsymbol{\| v}\| \mid=\left[\sum_{f}\left(\left\|\eta^{\frac{1}{2}} \boldsymbol{v}\right\|_{0, f}^{2}+\left\|\eta^{-\frac{1}{2}} \sigma(\boldsymbol{v}) \boldsymbol{n}\right\|_{0, f}^{2}\right)\right]^{1 / 2} .
$$


Since $\|\eta\|_{\infty}<C \omega$ and $\left\|\eta^{-1}\right\|_{\infty}<C \omega^{-1}$,

$$
\|\boldsymbol{v}\| \leq C\left[\sum_{f}\left(\omega\|\boldsymbol{v}\|_{0, f}^{2}+\omega^{-1}\|\sigma(\boldsymbol{v}) \boldsymbol{n}\|_{0, f}^{2}\right)\right]^{1 / 2}
$$

and applying the trace inequality [3]

$$
\|\boldsymbol{v}\|_{0, f}^{2} \leq C\left(h_{K_{f}}\|\nabla \boldsymbol{v}\|_{0, K_{f}}^{2}+h_{K_{f}}^{-1}\|\boldsymbol{v}\|_{0, K_{f}}^{2}\right)
$$

where $K_{f}$ is an element with face $f$ to (3.41), we obtain the following estimate

$$
\|\boldsymbol{v}\| \mid \leq\left[C \sum_{f} h_{K_{f}} \omega^{-1}\|\nabla \sigma(\boldsymbol{v}) \boldsymbol{n}\|_{0, K_{f}}^{2}+h_{K_{f}}^{-1} \omega^{-1}\|\sigma(\boldsymbol{v}) \boldsymbol{n}\|_{0, K_{f}}^{2}+h_{K_{f}} \omega\|\nabla \boldsymbol{v}\|_{0, K_{f}}^{2}+h_{K_{f}}^{-1} \omega\|\boldsymbol{v}\|_{0, K_{f}}^{2}\right]^{1 / 2} .
$$

For $h$ small enough we can write further that

$$
\|\boldsymbol{v}\| \mid \leq\left[C \sum_{f} h_{K_{f}}^{-1} \omega^{-1}\|\nabla \sigma(\boldsymbol{v}) \boldsymbol{n}\|_{0, K_{f}}^{2}+h_{K_{f}}^{-1} \omega^{-1}\|\sigma(\boldsymbol{v}) \boldsymbol{n}\|_{0, K_{f}}^{2}+h_{K_{f}}^{-1} \omega\|\nabla \boldsymbol{v}\|_{0, K_{f}}^{2}+h_{K_{f}}^{-1} \omega\|\boldsymbol{v}\|_{0, K_{f}}^{2}\right]^{1 / 2} .
$$

Because $\sigma(\boldsymbol{v})=(\lambda \nabla \cdot \boldsymbol{v}) I_{d}+\mu\left(\nabla \boldsymbol{v}+(\nabla \boldsymbol{v})^{T}\right)$ i.e. it consists of first derivatives of $\boldsymbol{v}$, we can estimate the first and second norms on the right hand side above using $\mathrm{H}^{2}$-norm of $\boldsymbol{v}$. In addition, the last two terms can be written as the $\mathrm{H}^{1}$-norm. Hence, using the assumed quasi-uniformity of mesh, we obtain

$$
\|\boldsymbol{v}\| \mid \leq C h^{-1 / 2}\left[\omega^{-1}\|\boldsymbol{v}\|_{2, \Omega}^{2}+\omega\|\boldsymbol{v}\|_{1, \Omega}^{2}\right]^{1 / 2} .
$$

Then using the regularity estimates (3.37) and (3.38) in (3.42) we obtain the estimate

$$
\begin{aligned}
\|\| \boldsymbol{v} \| & \leq C h^{-\frac{1}{2}}\left[\omega\left(1+\omega+\omega^{-2}\right)^{2}+\omega^{-1}\left(1+\omega^{2}+\omega^{-2}\right)\right]^{1 / 2}\|\boldsymbol{\psi}\|_{0, \Omega} \\
& \leq C h^{-1 / 2}\left(\omega^{\frac{3}{2}}+\omega^{-\frac{5}{2}}\right)\|\boldsymbol{\psi}\|_{0, \Omega} .
\end{aligned}
$$

Using the estimate (3.43) in equation (3.40), and choosing $\boldsymbol{\psi}=\boldsymbol{w}$ the estimate in (3.39) follows.

We state the main theorem of this section: an error estimate in $\mathrm{L}^{2}(\Omega)$ ( $c f$. Thm. 4.1 in [4]).

Theorem 3.14. Let $\boldsymbol{u}_{h}$ denote the piecewise defined local solution of $\boldsymbol{u}$ given by (3.29). Suppose that the domain $\Omega$ is convex and polyhedral, and the mesh is regular and quasi-uniform. Then the following estimate holds

$$
\left\|\boldsymbol{u}-\boldsymbol{u}_{h}\right\|_{0, \Omega} \leq C h^{-1 / 2}\left(\omega^{\frac{3}{2}}+\omega^{-\frac{5}{2}}\right)\left\|\left(I-P^{h}\right) \mathcal{X}\right\|_{X} .
$$

Proof. We begin the proof by replacing $\boldsymbol{w}$ by $\boldsymbol{u}-\boldsymbol{u}_{h}$ in equation (3.39). Then

$$
\begin{aligned}
\left\|\boldsymbol{u}-\boldsymbol{u}_{h}\right\|_{0, \Omega}^{2} \leq & C h^{-1}\left(\omega^{\frac{3}{2}}+\omega^{-\frac{5}{2}}\right)^{2}\left[\sum_{f \in \Sigma}\left(\left\|\eta^{\frac{1}{2}} \llbracket \boldsymbol{u}-\boldsymbol{u}_{h} \rrbracket \boldsymbol{n}\right\|_{0, f}^{2}+\left\|\eta^{-\frac{1}{2}} \llbracket \sigma\left(\boldsymbol{u}-\boldsymbol{u}_{h}\right) \rrbracket\right\|_{0, f}^{2}\right)\right. \\
& \left.+\sum_{f \in \Gamma}\left(\left\|\eta^{-\frac{1}{2}} \sigma\left(\boldsymbol{u}-\boldsymbol{u}_{h}\right) \boldsymbol{n}+i \eta^{-\frac{1}{2}} \eta\left(\boldsymbol{u}-\boldsymbol{u}_{h}\right)\right\|_{0, f}^{2}\right)\right]
\end{aligned}
$$

Now using estimate (3.30) the claim follows. 


\subsection{Error estimates using the approximation properties of plane waves}

In this section we investigate the approximation by plane waves and write an explicit estimate for $\left\|\boldsymbol{u}-\boldsymbol{u}_{h}\right\|_{0, \Omega}$. We rely heavily on the work of Moiola et al. see references [14,24-26].

We assume that our mesh consists of regular and quasi-uniform tetrahedra. These elements obviously satisfy the assumptions from [26] which we now recall. Any other choice of elements that satisfy these assumptions are also allowable for the estimates in this section. In particular, we assume that each element $K_{k} \subset \mathbb{R}^{3}$ is star-shaped with respect to a point $x_{K_{k}}$ and there exists $\varrho \in(0,1 / 2]$ such that $B_{\varrho h_{K_{k}}}\left(x_{K_{k}}\right) \subseteq K_{k}$ where $h_{K_{k}}$ is the diameter of $K_{k}$ and $B_{\varrho h_{K_{k}}}\left(x_{K_{k}}\right)$ is the ball of radius $\varrho h_{K_{k}}$ centered at $x_{K_{k}}$. In addition, we require that there exists $0<\varrho_{0} \leq \varrho$ such that $K_{k}$ is star-shaped with respect to $B_{\varrho_{0} h_{K_{k}}}\left(x_{K_{k}}\right)$. Of course this assumption is satisfied for the elements $K_{k}$ in our regular finite element mesh.

We need to use the Helmholtz decomposition for the solution $\boldsymbol{u}$ of the Navier equation written as

$$
\boldsymbol{u}=\nabla A+\nabla \times \boldsymbol{B}
$$

where $A$ is a scalar field and $\boldsymbol{B}$ is a vector field such that $\nabla \cdot \boldsymbol{B}=0$. We note that

$$
\begin{aligned}
\nabla \cdot \boldsymbol{u} & =\nabla^{2} A, \\
\nabla \times \boldsymbol{u} & =\nabla \times \nabla \times \boldsymbol{B},
\end{aligned}
$$

since $\nabla \cdot \boldsymbol{B}=0$ and $\nabla \times(\nabla A)=0$.

The fields $A$ and $\boldsymbol{B}$ satisfy respectively the Helmholtz and Maxwell system:

$$
\begin{aligned}
\nabla^{2} A+\kappa_{P}^{2} A & =0, \\
\nabla \times \nabla \times \boldsymbol{B}-\kappa_{S}^{2} \boldsymbol{B} & =\mathbf{0} .
\end{aligned}
$$

Then it is easily verified that $\boldsymbol{u}$ given by (3.45) satisfies the Navier equation.

In particular, as noted in $[24,25]$, we may choose

$$
\begin{aligned}
& A=-\frac{(\lambda+2 \mu)}{\rho \omega^{2}} \nabla \cdot \boldsymbol{u}, \\
& \boldsymbol{B}=\frac{\mu}{\rho \omega^{2}} \nabla \times \boldsymbol{u} .
\end{aligned}
$$

We can then write equation (3.45) as

$$
\boldsymbol{u}=\nabla A+\nabla \times \boldsymbol{B}=-\frac{(\lambda+2 \mu)}{\rho \omega^{2}} \nabla \nabla \cdot \boldsymbol{u}+\frac{\mu}{\rho \omega^{2}} \nabla \times \nabla \times \boldsymbol{u} .
$$

We will use this Helmholtz decomposition to write an estimate for $\left\|\mathcal{X}-\mathcal{Z}^{h}\right\|_{\mathrm{L}_{\eta}^{2}\left(\partial K_{k}\right)}$ for a suitable $\mathcal{Z}^{h} \in X^{h}$. Of course,

$$
\left\|\mathcal{X}-\mathcal{Z}^{h}\right\|_{X}^{2}=\frac{1}{2} \sum_{k=1}^{N}\left\|\mathcal{X}-\mathcal{Z}^{h}\right\|_{\mathrm{L}_{\eta}^{2}\left(\partial K_{k}\right)}^{2}
$$

Recall that $\mathcal{X}=(-\sigma(\boldsymbol{u}) \boldsymbol{n}-i \eta \boldsymbol{u})$ and

$$
\mathcal{Z}_{k}^{h}=\sum_{\ell=1}^{p_{k}^{P}} \mathcal{Z}_{k, \ell}^{P}\left(-\sigma\left(\boldsymbol{\phi}_{k, \ell}^{P}\right) \boldsymbol{n}_{k}-i \eta \boldsymbol{\phi}_{k, \ell}^{P}\right)+\sum_{\ell=1}^{p_{k}^{S}} \mathcal{Z}_{k, \ell}^{S H}\left(-\sigma\left(\boldsymbol{\phi}_{k, \ell}^{S H}\right) \boldsymbol{n}_{k}-i \eta \boldsymbol{\phi}_{k, \ell}^{S H}\right)+\sum_{\ell=1}^{p_{k}^{S}} \mathcal{Z}_{k, \ell}^{S V}\left(-\sigma\left(\boldsymbol{\phi}_{k, \ell}^{S V}\right) \boldsymbol{n}_{k}-i \eta \boldsymbol{\phi}_{k, \ell}^{S V}\right)
$$

The next theorem is a slight generalization of the estimates of $[24,25]$ to allow for different number of basis functions for $A$ and $\boldsymbol{B}$. Since the wave numbers in (3.48) and (3.49) differ, having the flexibility of choosing different bases for the two fields is very desirable [16]. 
We define $\kappa$-weighted norm $\|\boldsymbol{u}\|_{r, \kappa, K_{k}}$ for $\kappa>0$ by

$$
\|\boldsymbol{u}\|_{r, \kappa, K_{k}}:=\left(\sum_{j=0}^{r} \kappa^{2(r-j)}|\boldsymbol{u}|_{j, K_{k}}^{2}\right)^{\frac{1}{2}}
$$

where the seminorm is given by

$$
|\boldsymbol{u}|_{\alpha, K_{k}}^{2}=\sum_{\substack{i, j, l \\ i+j+l=\alpha}}\left\|\frac{\partial^{i}}{\partial x_{1}^{i}} \frac{\partial^{j}}{\partial x_{2}^{j}} \frac{\partial^{l}}{\partial x_{3}^{l}} \boldsymbol{u}\right\|_{0, K_{k}}^{2}
$$

where $i, j, l$ and $\alpha \in \mathbb{N}_{0}$.

Theorem 3.15. Suppose that $\boldsymbol{u}$ is given by (3.45) with $A \in \mathrm{H}^{r_{P}+1}\left(K_{k}\right), \boldsymbol{B} \in \mathrm{H}^{r_{S}+1}\left(K_{k}\right)^{3}$ and $\mathcal{X}=(-\sigma(\boldsymbol{u}) \boldsymbol{n}-$ $i \eta \boldsymbol{u})$. Let $q_{P} \geq 2 r_{P}+1, q_{S} \geq 2 r_{S}+1$ and suppose that $q_{P} \geq 2\left(1+2^{1 / \Lambda}\right), q_{S} \geq 2\left(1+2^{1 / \Lambda}\right)$ where $r_{P}, r_{S} \in \mathbb{N}$, the constant $\Lambda>0$ depends only on the shape of the element $K_{k}$, see Theorem 3.2. in [26]. Then, there exists $\mathcal{Z}^{h} \in X^{h}$ such that

$$
\begin{aligned}
\left\|\mathcal{X}-\mathcal{Z}^{h}\right\|_{L_{\eta}^{2}\left(\partial K_{k}\right)} \leq & C\left\|\eta^{-\frac{1}{2}}\right\|_{\infty}\left[h_{K_{k}}^{r_{P}-\frac{3}{2}} q_{P}^{-\Lambda\left(r_{P}-\frac{3}{2}\right)}\left(\|\eta\|_{\infty} \alpha+\beta\right)\|A\|_{r_{P}+1, \kappa_{P}, K_{k}}\right. \\
& \left.+h_{K_{k}}^{r_{S}-\frac{3}{2}} q_{S}^{-\Lambda\left(r_{S}-\frac{3}{2}\right)}\left(\|\eta\|_{\infty} \gamma+\delta\right)\|\boldsymbol{B}\|_{r_{S}+1, \kappa_{S}, K_{k}}\right]
\end{aligned}
$$

where

$$
\begin{aligned}
& \alpha=h_{K_{k}} q_{P}^{-\Lambda}\left(1+\left(\kappa_{P} h_{K_{k}}\right)^{q_{P}-r_{P}+\frac{19}{2}}\right) \exp \left(\left(\frac{7}{4}-\frac{3}{4} \varrho\right) \kappa_{P} h_{K_{k}}\right), \\
& \beta=\left(1+\left(\kappa_{P} h_{K_{k}}\right)^{q_{P}-r_{P}+\frac{21}{2}}\right) \exp \left(\left(\frac{7}{4}-\frac{3}{4} \varrho\right) \kappa_{P} h_{K_{k}}\right), \\
& \gamma=h_{K_{k}} q_{S}^{-\Lambda}\left(1+\left(\kappa_{S} h_{K_{k}}\right)^{q_{S}-r_{S}+\frac{19}{2}}\right) \exp \left(\left(\frac{7}{4}-\frac{3}{4} \varrho\right) \kappa_{S} h_{K_{k}}\right), \\
& \delta=\left(1+\left(\kappa_{S} h_{K_{k}}\right)^{q_{S}-r_{S}+\frac{21}{2}}\right) \exp \left(\left(\frac{7}{4}-\frac{3}{4} \varrho\right) \kappa_{S} h_{K_{k}}\right) .
\end{aligned}
$$

Remark 3.16. In the proof we will use parameters $M_{P}$ and $M_{S}$ which are determined from the set of directions of the plane waves on each element. In [24-26] Hiptmair et al. show that in the best case $M_{P}=\mathcal{O}\left(p^{P}\right)=\mathcal{O}\left(q_{P}^{2}\right)$, and for their directions, see [30,32], (which we also use) $M_{P}=\mathcal{O}\left(p^{P} q_{P}\right)=\mathcal{O}\left(q_{P}^{3}\right)$. For our theory it suffices that

$$
M_{P}=\mathcal{O}\left(q_{P}^{\alpha}\right), \quad M_{S}=\mathcal{O}\left(q_{S}^{\alpha}\right),
$$

for some $\alpha>0$ fixed, and we assume this from now on. Note that the number of P-wave basis functions is $p^{P}=\left(q_{P}+1\right)^{2}$, the number of SH-wave and SV-wave basis functions is each $p^{S}=\left(q_{S}+1\right)^{2}$.

Proof. Our proof follows the techniques from [14,24,25]. On $K_{k}$ any function $\mathcal{Z}^{h} \in X^{h}$ can be written as

$$
\mathcal{Z}^{h}=-\sigma\left(\nabla A_{h}+\nabla \times \boldsymbol{B}_{h}\right)-i \eta\left(\nabla A_{h}+\nabla \times \boldsymbol{B}_{h}\right)
$$

for some $A_{h}$ and $\boldsymbol{B}_{h}$ given by

$$
A_{h}=\sum_{\ell=1}^{p^{P}} \alpha_{\ell}^{P} \exp \left(i \kappa_{P} \boldsymbol{a}_{\ell} \cdot \boldsymbol{x}\right) \quad \text { and } \quad \boldsymbol{B}_{h}=\sum_{\ell=1}^{p^{S}} \sum_{n=1}^{3} \alpha_{\ell}^{n} \boldsymbol{\beta}_{\ell}^{n},
$$


where we have suppressed the dependence on $k$ and where

$$
\boldsymbol{\beta}_{\ell}^{1}=\boldsymbol{a}_{\ell}^{S \perp} \exp \left(i \kappa_{S} \boldsymbol{a}_{\ell}^{S} \cdot \boldsymbol{x}\right), \quad \boldsymbol{\beta}_{\ell}^{2}=\boldsymbol{a}_{\ell}^{S \perp} \times \boldsymbol{a}_{\ell}^{S} \exp \left(i \kappa_{S} \boldsymbol{a}_{\ell}^{S} \cdot \boldsymbol{x}\right) \quad \text { and } \quad \boldsymbol{\beta}_{\ell}^{3}=\boldsymbol{a}_{\ell}^{S} \exp \left(i \kappa_{S} \boldsymbol{a}_{\ell}^{S} \cdot \boldsymbol{x}\right) .
$$

To see this, we note that as in [14]

$$
\nabla \times \boldsymbol{\beta}_{\ell}^{1}=-i \kappa_{S} \boldsymbol{\beta}_{\ell}^{2}, \quad \nabla \times \boldsymbol{\beta}_{\ell}^{2}=i \kappa_{S} \boldsymbol{\beta}_{\ell}^{1} \quad \text { and } \quad \nabla \times \boldsymbol{\beta}_{\ell}^{3}=0 .
$$

Then we can write

$$
\begin{aligned}
\left\|\mathcal{X}-\mathcal{Z}^{h}\right\|_{L_{\eta}^{2}\left(\partial K_{k}\right)} \leq & \left\|\eta^{-\frac{1}{2}}\right\|_{\infty}\left\|-\sigma(\boldsymbol{u}) \boldsymbol{n}-i \eta \boldsymbol{u}-\left(-\sigma\left(\boldsymbol{u}_{h}\right) \boldsymbol{n}-i \eta \boldsymbol{u}_{h}\right)\right\|_{0, \partial K_{k}} \\
= & \left\|\eta^{-\frac{1}{2}}\right\|_{\infty} \|-\sigma(\nabla A+\nabla \times \boldsymbol{B}) \boldsymbol{n}-i \eta(\nabla A+\nabla \times \boldsymbol{B}) \\
& -\left(-\sigma\left(\nabla A_{h}+\nabla \times \boldsymbol{B}_{h}\right) \boldsymbol{n}-i \eta\left(\nabla A_{h}+\nabla \times \boldsymbol{B}_{h}\right)\right) \|_{0, \partial K_{k}} .
\end{aligned}
$$

Rearranging terms and applying triangle inequality we obtain

$$
\begin{aligned}
\left\|\mathcal{X}-\mathcal{Z}^{h}\right\|_{L_{\eta}^{2}\left(\partial K_{k}\right)} \leq & \left\|\eta^{-\frac{1}{2}}\right\|_{\infty}\left[\left\|-\sigma\left(\nabla\left(A-A_{h}\right)\right) \boldsymbol{n}-i \eta\left(\nabla\left(A-A_{h}\right)\right)\right\|_{0, \partial K_{k}}\right. \\
& \left.+\left\|-\sigma\left(\nabla \times\left(\boldsymbol{B}-\boldsymbol{B}_{h}\right)\right) \boldsymbol{n}-i \eta\left(\nabla \times\left(\boldsymbol{B}-\boldsymbol{B}_{h}\right)\right)\right\|_{0, \partial K_{k}}\right] .
\end{aligned}
$$

We start by investigating in detail the first term on the right hand side of above equation. The trace estimate can now be written as follows $[3,13,14]$

$$
\begin{aligned}
\left\|\sigma\left(\nabla\left(A-A_{h}\right)\right) \boldsymbol{n}\right\|_{0, \partial K_{k}}^{2} \leq & C\left(h_{K_{k}}^{-1}\left\|\sigma\left(\nabla\left(A-A_{h}\right)\right)\right\|_{0, K_{k}}^{2}\right. \\
& \left.+\left\|\sigma\left(\nabla\left(A-A_{h}\right)\right)\right\|_{0, K_{k}}\left\|\nabla \sigma\left(\nabla\left(A-A_{h}\right)\right)\right\|_{0, K_{k}}\right) .
\end{aligned}
$$

Hence, we can estimate

$$
\begin{aligned}
& \left\|\sigma\left(\nabla\left(A-A_{h}\right)\right)\right\|_{0, K_{k}} \leq C\left|A-A_{h}\right|_{2, K_{k}}, \\
& \left\|\nabla \sigma\left(\nabla\left(A-A_{h}\right)\right)\right\|_{0, K_{k}} \leq C\left|A-A_{h}\right|_{3, K_{k}} .
\end{aligned}
$$

We note that

$$
|\boldsymbol{u}|_{r, K_{k}} \leq\|\boldsymbol{u}\|_{r, \kappa, K_{k}} .
$$

In a similar manner as earlier we can write

$$
\left\|\nabla\left(A-A_{h}\right)\right\|_{0, \partial K_{k}}^{2} \leq C\left(h_{K_{k}}^{-1}\left\|\nabla\left(A-A_{h}\right)\right\|_{0, K_{k}}^{2}+\left\|\nabla\left(A-A_{h}\right)\right\|_{0, K_{k}}\left\|\nabla \nabla\left(A-A_{h}\right)\right\|_{0, K_{k}}\right) .
$$

Moreover,

$$
\begin{aligned}
\left\|\nabla\left(A-A_{h}\right)\right\|_{0, K_{k}} & \leq C\left|A-A_{h}\right|_{1, K_{k}}, \\
\left\|\nabla \nabla\left(A-A_{h}\right)\right\|_{0, K_{k}} & \leq C\left|A-A_{h}\right|_{2, K_{k}} .
\end{aligned}
$$

Now using (3.54) and (3.57) together with (3.55), (3.56), (3.58) and (3.59) as follows

$$
\begin{aligned}
\left\|-\sigma\left(\nabla\left(A-A_{h}\right)\right) \boldsymbol{n}-i \eta\left(\nabla\left(A-A_{h}\right)\right)\right\|_{0, \partial K_{k}} \leq & \left\|\sigma\left(\nabla\left(A-A_{h}\right)\right) \boldsymbol{n}\right\|_{0, \partial K_{k}}+\|\eta\|_{\infty}\left\|\nabla\left(A-A_{h}\right)\right\|_{0, \partial K_{k}} \\
\leq & C\left(\|\eta\|_{\infty} h_{K_{k}}^{-1}\left|A-A_{h}\right|_{1, K_{k}}^{2}+h_{K_{k}}^{-1}\left|A-A_{h}\right|_{2, K_{k}}^{2}\right. \\
& \left.+\left|A-A_{h}\right|_{2, K_{k}}\left|A-A_{h}\right|_{3, K_{k}}+\|\eta\|_{\infty}\left|A-A_{h}\right|_{1, K_{k}}\left|A-A_{h}\right|_{2, K_{k}}\right)^{1 / 2}
\end{aligned}
$$


From [14] we can choose $\alpha_{\ell}^{P}, 1 \leq \ell \leq p^{P}$, and $q_{P} \geq 2 r_{P}+1$, such that we can find the bounds for every $0 \leq j \leq r_{P}$ as follows

$$
\left\|A-\sum_{\ell=1}^{p^{P}} \alpha_{\ell}^{P} \exp \left(i \kappa_{P} \boldsymbol{a}_{\ell} \cdot \boldsymbol{x}\right)\right\|_{j, \kappa_{P}, K_{k}} \leq C h_{K_{k}}^{r_{P}+1-j}\left(1+\left(\kappa_{P} h_{K_{k}}\right)^{q_{P}+j-r_{P}+8}\right) \Theta_{P}(j)\|A\|_{r_{P}+1, \kappa_{P}, K_{k}},
$$

where

$$
\Theta_{P}(j):=\left(q_{P}^{-\Lambda\left(r_{P}+1-j\right)}+\left(\varrho q_{P}\right)^{-\frac{q_{P}-3}{2}} M_{P}\right) \exp \left(\left(\frac{7}{4}-\frac{3}{4} \varrho\right) \kappa_{P} h_{K_{k}}\right) .
$$

Then using the fact that

$$
\left(1+\left(\kappa_{P} h_{K_{k}}\right)^{x}\right)\left(1+\left(\kappa_{P} h_{K_{k}}\right)^{y}\right) \leq C\left(1+\left(\kappa_{P} h_{K_{k}}\right)^{x+y}\right)
$$

for any $x>0$ and $y>0$, we have

$$
\begin{aligned}
\|-\sigma( & \left.\nabla\left(A-A_{h}\right)\right) \boldsymbol{n}-i \eta\left(\nabla\left(A-A_{h}\right)\right) \|_{0, \partial K_{k}} \\
\leq & C\left[\|\eta\|_{\infty} h_{K_{k}}^{r_{P}-\frac{1}{2}}\left(\left(1+\left(\kappa_{P} h_{K_{k}}\right)^{q_{P}-r_{P}+9}\right) \Theta_{P}(1)+\left(1+\left(\kappa_{P} h_{K_{k}}\right)^{q_{P}-r_{P}+\frac{19}{2}}\right) \sqrt{\Theta_{P}(1) \Theta_{P}(2)}\right)\right. \\
& \left.+h_{K_{k}}^{r_{P}-\frac{3}{2}}\left(\left(1+\left(\kappa_{P} h_{K_{k}}\right)^{q_{P}-r_{P}+10}\right) \Theta_{P}(2)+\left(1+\left(\kappa_{P} h_{K_{k}}\right)^{q_{P}-r_{P}+\frac{21}{2}}\right) \sqrt{\Theta_{P}(2) \Theta_{P}(3)}\right)\right]\|A\|_{r_{P}+1, \kappa_{P}, K_{k}} .
\end{aligned}
$$

Since $0<\Lambda<1, \Lambda r_{p}$ increases more slowly than $q_{P} / 2$. Under our assumption on $M_{P}$ we see that for $r_{P}$ large enough

$$
\Theta_{P}(j) \leq C q_{P}^{-\Lambda\left(r_{P}+1-j\right)} \exp \left(\left(\frac{7}{4}-\frac{3}{4} \varrho\right) \kappa_{P} h_{h_{k}}\right), \quad 1 \leq j \leq 3
$$

so

$$
\sqrt{\Theta_{P}(j) \Theta_{P}(j+1)} \leq C q_{P}^{-\Lambda\left(r_{P}-j+\frac{1}{2}\right)} \exp \left(\left(\frac{7}{4}-\frac{3}{4} \varrho\right) \kappa_{P} h_{K_{k}}\right)
$$

Thus

$$
\left\|-\sigma\left(\nabla\left(A-A_{h}\right)\right) \boldsymbol{n}-i \eta\left(\nabla\left(A-A_{h}\right)\right)\right\|_{0, \partial K_{k}} \leq C h_{K_{k}}^{r_{P}-\frac{3}{2}} q_{P}^{-\Lambda\left(r_{P}-\frac{3}{2}\right)}\left(\|\eta\|_{\infty} \alpha+\beta\right)\|A\|_{r_{P}+1, \kappa_{P}, K_{k}},
$$

where

$$
\begin{aligned}
& \alpha=h_{K_{k}} q_{P}^{-\Lambda}\left(1+\left(\kappa_{P} h_{K_{k}}\right)^{q_{P}-r_{P}+\frac{19}{2}}\right) \exp \left(\left(\frac{7}{4}-\frac{3}{4} \varrho\right) \kappa_{P} h_{K_{k}}\right), \\
& \beta=\left(1+\left(\kappa_{P} h_{K_{k}}\right)^{q_{P}-r_{P}+\frac{21}{2}}\right) \exp \left(\left(\frac{7}{4}-\frac{3}{4} \varrho\right) \kappa_{P} h_{K_{k}}\right) .
\end{aligned}
$$

We now want to estimate $\left\|-\sigma\left(\nabla \times\left(\boldsymbol{B}-\boldsymbol{B}_{h}\right)\right) \boldsymbol{n}-i \eta\left(\nabla \times\left(\boldsymbol{B}-\boldsymbol{B}_{h}\right)\right)\right\|_{0, \partial K_{k}}$. The trace estimate gives

$$
\begin{aligned}
\left\|\sigma\left(\nabla \times\left(\boldsymbol{B}-\boldsymbol{B}_{h}\right)\right) \boldsymbol{n}\right\|_{0, \partial K_{k}}^{2} \leq & C\left(h_{K_{k}}^{-1}\left\|\sigma\left(\nabla \times\left(\boldsymbol{B}-\boldsymbol{B}_{h}\right)\right)\right\|_{0, K_{k}}^{2},\right. \\
& \left.+\left\|\sigma\left(\nabla \times\left(\boldsymbol{B}-\boldsymbol{B}_{h}\right)\right)\right\|_{0, K_{k}}\left\|\nabla \sigma\left(\nabla \times\left(\boldsymbol{B}-\boldsymbol{B}_{h}\right)\right)\right\|_{0, K_{k}}\right) \\
\left\|\nabla \times\left(\boldsymbol{B}-\boldsymbol{B}_{h}\right)\right\|_{0, \partial K_{k}}^{2} \leq & C\left(h_{K_{k}}^{-1}\left\|\nabla \times\left(\boldsymbol{B}-\boldsymbol{B}_{h}\right)\right\|_{0, K_{k}}^{2}\right. \\
& \left.+\left\|\nabla \times\left(\boldsymbol{B}-\boldsymbol{B}_{h}\right)\right\|_{0, K_{k}}\left\|\nabla \nabla \times\left(\boldsymbol{B}-\boldsymbol{B}_{h}\right)\right\|_{0, K_{k}}\right) .
\end{aligned}
$$


Furthermore, we can estimate

$$
\begin{aligned}
\left\|\sigma\left(\nabla \times\left(\boldsymbol{B}-\boldsymbol{B}_{h}\right)\right)\right\|_{0, K_{k}} & \leq C\left|\nabla \times\left(\boldsymbol{B}-\boldsymbol{B}_{h}\right)\right|_{1, K_{k}}, \\
\left\|\nabla \sigma\left(\nabla \times\left(\boldsymbol{B}-\boldsymbol{B}_{h}\right)\right)\right\|_{0, K_{k}} & \leq C\left|\nabla \times\left(\boldsymbol{B}-\boldsymbol{B}_{h}\right)\right|_{2, K_{k}}, \\
\left\|\nabla \times\left(\boldsymbol{B}-\boldsymbol{B}_{h}\right)\right\|_{0, K_{k}} & \leq C\left|\nabla \times\left(\boldsymbol{B}-\boldsymbol{B}_{h}\right)\right|_{0, K_{k}}, \\
\left\|\nabla \nabla \times\left(\boldsymbol{B}-\boldsymbol{B}_{h}\right)\right\|_{0, K_{k}} & \leq C\left|\nabla \times\left(\boldsymbol{B}-\boldsymbol{B}_{h}\right)\right|_{1, K_{k}} .
\end{aligned}
$$

Thus, we can write

$$
\begin{aligned}
&\left\|-\sigma\left(\nabla \times\left(\boldsymbol{B}-\boldsymbol{B}_{h}\right)\right) \boldsymbol{n}-i \eta\left(\nabla \times\left(\boldsymbol{B}-\boldsymbol{B}_{h}\right)\right)\right\|_{0, \partial K_{k}} \\
& \leq\left\|-\sigma\left(\nabla \times\left(\boldsymbol{B}-\boldsymbol{B}_{h}\right)\right) \boldsymbol{n}\right\|_{0, \partial K_{k}}+\left\|-i \eta\left(\nabla \times\left(\boldsymbol{B}-\boldsymbol{B}_{h}\right)\right)\right\|_{0, \partial K_{k}} \\
& \leq C\left(h_{K_{k}}^{-1}\left|\nabla \times\left(\boldsymbol{B}-\boldsymbol{B}_{h}\right)\right|_{1, K_{k}}^{2}+\left|\nabla \times\left(\boldsymbol{B}-\boldsymbol{B}_{h}\right)\right|_{1, K_{k}}\left|\nabla \times\left(\boldsymbol{B}-\boldsymbol{B}_{h}\right)\right|_{2, K_{k}}\right. \\
&\left.+h_{K_{k}}^{-1}\|\eta\|_{\infty}\left|\nabla \times\left(\boldsymbol{B}-\boldsymbol{B}_{h}\right)\right|_{0, K_{k}}^{2}+\|\eta\|_{\infty}\left|\nabla \times\left(\boldsymbol{B}-\boldsymbol{B}_{h}\right)\right|_{0, K_{k}}\left|\nabla \times\left(\boldsymbol{B}-\boldsymbol{B}_{h}\right)\right|_{1, K_{k}}\right)^{\frac{1}{2}} .
\end{aligned}
$$

As in [25] we have

$$
\begin{aligned}
\left\|\nabla \times\left(\boldsymbol{B}-\boldsymbol{B}_{h}\right)\right\|_{j-1, \kappa_{S}, K_{k}} & =\left\|\nabla \times \boldsymbol{B}-i \kappa_{S} \sum_{\ell=1}^{p^{S}}\left(-\alpha_{\ell}^{S V} \boldsymbol{\beta}_{\ell}^{S H}+\alpha_{\ell}^{S H} \boldsymbol{\beta}_{\ell}^{S V}\right)\right\|_{j-1, \kappa_{S}, K_{k}} \\
& =\left\|\nabla \times \boldsymbol{B}-\sum_{\ell=1}^{p^{S}} \sum_{n=1}^{3} \alpha_{\ell}^{n} \nabla \times \boldsymbol{\beta}_{\ell}^{n}\right\|_{j-1, \kappa_{S}, K_{k}} \\
& =\left\|\nabla \times\left(\boldsymbol{B}-\sum_{\ell=1}^{p^{S}} \sum_{n=1}^{3} \alpha_{\ell}^{n} \boldsymbol{\beta}_{\ell}^{n}\right)\right\|_{j-1, \kappa_{S}, K_{k}} \\
& \leq\left\|\boldsymbol{B}-\sum_{\ell=1}^{p^{S}} \sum_{n=1}^{3} \alpha_{\ell}^{n} \boldsymbol{\beta}_{\ell}^{n}\right\|_{j, \kappa_{S}, K_{k}} .
\end{aligned}
$$

Again we fix $q_{S}$ such that $q_{S} \geq 2 r_{S}+1$ and $q_{S} \geq 2\left(1+2^{1 / \Lambda}\right)$. From [14] we can choose $\alpha_{\ell}^{n}, 1 \leq n \leq 3,1 \leq \ell \leq p^{S}$, of $\boldsymbol{B}_{h}$ such that the following bound holds

$$
\left\|\boldsymbol{B}-\sum_{\ell=1}^{p^{S}} \sum_{n=1}^{3} \alpha_{\ell}^{n} \boldsymbol{\beta}_{\ell}^{n}\right\|_{j, \kappa_{S}, K_{k}} \leq C h_{K_{k}}^{r_{S}+1-j}\left(1+\left(\kappa_{S} h_{K_{k}}\right)^{q_{S}+j-r_{S}+8}\right) \Theta_{S}(j)\|\boldsymbol{B}\|_{r_{S}+1, \kappa_{S}, K_{k}}
$$

where

$$
\Theta_{S}(j)=\left[q_{S}^{\Lambda\left(r_{S}+1-j\right)}+\left(\varrho q_{S}\right)^{-\frac{q_{S}-3}{2}} M_{S}\right] \exp \left(\left(\frac{7}{4}-\frac{3}{4} \varrho\right) \kappa_{S} h_{K_{k}}\right)
$$

Hence,

$$
\left\|\nabla \times\left(\boldsymbol{B}-\boldsymbol{B}_{h}\right)\right\|_{j-1, \kappa_{S}, K_{k}} \leq C h_{K_{k}}^{r_{S}+1-j}\left(1+\left(\kappa_{S} h_{K_{k}}\right)^{q_{S}+j-r_{S}+8}\right) \Theta_{S}(j)\|\boldsymbol{B}\|_{r_{S}+1, \kappa_{S}, K_{k}}
$$


We investigate equation (3.62) with $j=1,2$ and 3 similar manner as earlier. Using these estimates we obtain

$$
\begin{aligned}
\|-\sigma(\nabla & \left.\times\left(\boldsymbol{B}-\boldsymbol{B}_{h}\right)\right) \boldsymbol{n}-i \eta\left(\nabla \times\left(\boldsymbol{B}-\boldsymbol{B}_{h}\right)\right) \|_{0, \partial K_{k}} \\
\leq & C\left(h_{K_{k}}^{-1}\left|\nabla \times\left(\boldsymbol{B}-\boldsymbol{B}_{h}\right)\right|_{1, K_{k}}^{2}+\left|\nabla \times\left(\boldsymbol{B}-\boldsymbol{B}_{h}\right)\right|_{1, K_{k}}\left|\nabla \times\left(\boldsymbol{B}-\boldsymbol{B}_{h}\right)\right|_{2, K_{k}}\right. \\
& \left.+h_{K_{k}}^{-1}\|\eta\|_{\infty}\left|\nabla \times\left(\boldsymbol{B}-\boldsymbol{B}_{h}\right)\right|_{0, K_{k}}^{2}+\|\eta\|_{\infty}\left|\nabla \times\left(\boldsymbol{B}-\boldsymbol{B}_{h}\right)\right|_{0, K_{k}}\left|\nabla \times\left(\boldsymbol{B}-\boldsymbol{B}_{h}\right)\right|_{1, K_{k}}\right)^{\frac{1}{2}} \\
\leq & C\left(h_{K_{k}}^{r_{S}-\frac{3}{2}}\left(\left(1+\left(\kappa_{S} h_{K_{k}}\right)^{q_{S}-r_{s}+10}\right) \Theta_{S}(2)+\left(1+\left(\kappa_{S} h_{K_{k}}\right)^{q_{S}-r_{S}+\frac{21}{2}}\right) \sqrt{\Theta_{S}(2) \Theta_{S}(3)}\right)\right. \\
& \left.+\|\eta\|_{\infty} h_{K_{k}}^{r_{S}-\frac{1}{2}}\left(\left(1+\left(\kappa_{S} h_{K_{k}}\right)^{q_{S}-r_{s}+9}\right) \Theta_{S}(1)+\left(1+\left(\kappa_{S} h_{K_{k}}\right)^{q_{S}-r_{S}+\frac{19}{2}}\right) \sqrt{\Theta_{S}(1) \Theta_{S}(2)}\right)\right) \\
& \cdot\|\boldsymbol{B}\|_{r_{S}+1, \kappa_{S}, K_{k}} .
\end{aligned}
$$

Using the same arguments used when deriving (3.61) we have

$$
\left\|-\sigma\left(\nabla \times\left(\boldsymbol{B}-\boldsymbol{B}_{h}\right)\right) \boldsymbol{n}-i \eta\left(\nabla \times\left(\boldsymbol{B}-\boldsymbol{B}_{h}\right)\right)\right\|_{0, \partial K_{k}} \leq C h_{K_{k}}^{r_{S}-\frac{3}{2}} q_{S}^{-\Lambda\left(r_{S}-\frac{3}{2}\right)}\left(\|\eta\|_{\infty} \gamma+\delta\right)\|\boldsymbol{B}\|_{r_{S}+1, \kappa_{S}, K_{k}},
$$

where

$$
\begin{aligned}
& \gamma=h_{K_{k}} q_{S}^{-\Lambda}\left(1+\left(\kappa_{S} h_{K_{k}}\right)^{q_{S}-r_{S}+\frac{19}{2}}\right) \exp \left(\left(\frac{7}{4}-\frac{3}{4} \varrho\right) \kappa_{S} h_{K_{k}}\right), \\
& \delta=\left(1+\left(\kappa_{S} h_{K_{k}}\right)^{q_{S}-r_{S}+\frac{21}{2}}\right) \exp \left(\left(\frac{7}{4}-\frac{3}{4} \varrho\right) \kappa_{S} h_{K_{k}}\right) .
\end{aligned}
$$

Combining equations (3.61) and (3.63) we obtain the desired estimate.

Using Theorem 3.15 element by element we obtain the following global result.

Corollary 3.17. Under the assumptions of Theorem 3.15, suppose $q_{P}$ and $q_{S}$ are identical on all elements, then

$$
\begin{aligned}
\left\|\mathcal{X}-\mathcal{Z}^{h}\right\|_{X} \leq & C\left\|\eta^{-\frac{1}{2}}\right\|_{\infty}\left[h^{r_{P}-\frac{3}{2}} q_{P}^{-\Lambda\left(r_{P}-\frac{3}{2}\right)}\left(\|\eta\|_{\infty} \alpha+\beta\right)\|A\|_{r_{P}+1, \kappa_{P}, \Omega}\right. \\
& \left.+h^{r_{S}-\frac{3}{2}} q_{S}^{-\Lambda\left(r_{S}-\frac{3}{2}\right)}\left(\|\eta\|_{\infty} \gamma+\delta\right)\|\boldsymbol{B}\|_{r_{S}+1, \kappa_{S}, \Omega}\right] .
\end{aligned}
$$

Now we can write our main convergence result.

Theorem 3.18. Under the conditions of Theorem 3.15, assume also that $\Omega$ is a convex polyhedron and $q_{P}$ and $q_{S}$ are identical on all elements. In addition, assume that the mesh is regular and quasi-uniform. Then the following estimate holds:

$$
\begin{aligned}
\left\|\boldsymbol{u}-\boldsymbol{u}_{h}\right\|_{0, \Omega} \leq & C\left(\omega^{-\frac{1}{2}}+\omega^{-\frac{9}{2}}\right)\left[h^{r_{P}-2} q_{P}^{-\Lambda\left(r_{P}-\frac{3}{2}\right)}\left(\omega^{\frac{1}{2}} \alpha+\omega^{-\frac{1}{2}} \beta\right)\|\nabla \cdot \boldsymbol{u}\|_{r_{P}+1, \kappa_{P}, \Omega}\right. \\
& \left.+h^{r_{S}-2} q_{S}^{-\Lambda\left(r_{S}-\frac{3}{2}\right)}\left(\omega^{\frac{1}{2}} \gamma+\omega^{-\frac{1}{2}} \delta\right)\|\nabla \times \boldsymbol{u}\|_{r_{S}+1, \kappa_{S}, \Omega}\right]
\end{aligned}
$$

where $C$ depends on $\rho, \mu$ and $\lambda$.

Proof. The proof is straightforward using (3.64) in equation (3.44). Thus we have

$$
\begin{aligned}
\left\|\boldsymbol{u}-\boldsymbol{u}_{h}\right\|_{0, \Omega} \leq & C h^{-1 / 2}\left(\omega^{\frac{3}{2}}+\omega^{-\frac{5}{2}}\right)\left\|\eta^{-\frac{1}{2}}\right\|_{\infty}\left[h^{r_{P}-\frac{3}{2}} q_{P}^{-\Lambda\left(r_{P}-\frac{3}{2}\right)}\left(\|\eta\|_{\infty} \alpha+\beta\right)\|A\|_{r_{P}+1, \kappa_{P}, \Omega}\right. \\
& \left.+h^{r_{S}-\frac{3}{2}} q_{S}^{-\Lambda\left(r_{S}-\frac{3}{2}\right)}\left(\|\eta\|_{\infty} \gamma+\delta\right)\|\boldsymbol{B}\|_{r_{S}+1, \kappa_{S}, \Omega}\right] .
\end{aligned}
$$


Taking into account the assumed bounds on $\|\eta\|_{\infty}$ and $\left\|\eta^{-1}\right\|_{\infty}$ and equations (3.50) and (3.51) we obtain the desired estimate:

$$
\begin{aligned}
\left\|\boldsymbol{u}-\boldsymbol{u}_{h}\right\|_{0, \Omega} \leq & C h^{-1 / 2}\left(\omega^{\frac{3}{2}}+\omega^{-\frac{5}{2}}\right)\left[h^{r_{P}-\frac{3}{2}} q_{P}^{-\Lambda\left(r_{P}-\frac{3}{2}\right)}\left(\omega^{\frac{1}{2}} \alpha+\omega^{-\frac{1}{2}} \beta\right) \omega^{-2}\|\nabla \cdot \boldsymbol{u}\|_{r_{P}+1, \kappa_{P}, \Omega}\right. \\
& \left.+h^{r_{S}-\frac{3}{2}} q_{S}^{-\Lambda\left(r_{S}-\frac{3}{2}\right)}\left(\omega^{\frac{1}{2}} \gamma+\omega^{-\frac{1}{2}} \delta\right) \omega^{-2}\|\nabla \times \boldsymbol{u}\|_{r_{S}+1, \kappa_{S}, \Omega}\right] .
\end{aligned}
$$

Corollary 3.19. Let $q_{P}, q_{S}$ be fixed, and assume that $\kappa_{S} h \leq C$ for some fixed $C$. Then

$$
\left\|\boldsymbol{u}-\boldsymbol{u}_{h}\right\|_{0, \Omega} \leq C\left(\omega^{-1}+\omega^{-5}\right)(1+\omega)\left(h^{r_{P}-2}\|\nabla \cdot \boldsymbol{u}\|_{r_{P}+1, \kappa_{P}, \Omega}+h^{r_{S}-2}\|\nabla \times \boldsymbol{u}\|_{r_{S}+1, \kappa_{S}, \Omega}\right) .
$$

Remark 3.20. The assumption $\kappa_{S} h \leq C$ is also necessary for a finite element method to converge under mesh refinement.

Proof. Equation (3.61) can be written as

$$
\left\|-\sigma\left(\nabla\left(A-A_{h}\right)\right) \boldsymbol{n}-i \eta\left(\nabla\left(A-A_{h}\right)\right)\right\|_{0, \partial K_{k}} \leq C h_{K_{k}}^{r_{P}-\frac{3}{2}}(1+\omega)\|A\|_{r_{P}+1, \kappa_{P}, K_{k}}
$$

since $q_{P}$ is fixed and $\|\eta\|_{\infty}=\mathcal{O}(\omega)$, and provided $\kappa_{P} h_{K_{k}} \leq C$. Similarly, $h_{K_{k}} \kappa_{S} \leq C$ for some $C$ and $\|\eta\|_{\infty}=$ $\mathcal{O}(\omega)$ and $q_{S}$ is fixed then equation (3.63) can be written as

$$
\left\|-\sigma\left(\nabla \times\left(\boldsymbol{B}-\boldsymbol{B}_{h}\right)\right) \boldsymbol{n}-i \eta\left(\nabla \times\left(\boldsymbol{B}-\boldsymbol{B}_{h}\right)\right)\right\|_{0, \partial K_{k}} \leq C h_{K_{k}}^{r_{S}-\frac{3}{2}}(1+\omega)\|\boldsymbol{B}\|_{r_{S}+1, \kappa_{S}, K_{k}} .
$$

Furthermore, we can write

$$
\left\|\mathcal{X}-\mathcal{Z}^{h}\right\|_{X} \leq C(1+\omega) \omega^{-\frac{1}{2}}\left(h^{r_{P}-\frac{3}{2}}\|A\|_{r_{P}+1, \kappa_{P}, \Omega}+h^{r_{S}-\frac{3}{2}}\|\boldsymbol{B}\|_{r_{S}+1, \kappa_{S}, \Omega}\right) .
$$

Then using (3.65) in (3.44) and taking into account (3.50) and (3.51) the claim follows.

\section{Numerical Results}

In this section we shall investigate the $p$-convergence and $h$-convergence of the UWVF for the Navier equation. Simulations are made in Matlab and the coefficient vector $\boldsymbol{x}$ in (2.27) is solved using the stabilized biconjugate gradient method (Matlab function: bicgstab). The maximum number of iterations is set 10000 and the residual limit is $10^{-6}$. To be consistent with the theory we choose directions in the elastic plane wave basis from [32]. In [21] the directions are chosen from [12] as in the 3D acoustic and electromagnetic UWVF, see [17, 18] respectively.

\subsection{Elastic plane wave propagation in a cube}

To be consistent with the error estimates, derived previously, we choose the Robin boundary condition $Q=0$ in equation (2.2). We shall consider a simple model problem as shown in [21]. The exact solution is

$$
\begin{aligned}
\boldsymbol{u} & =\boldsymbol{u}_{P}+\boldsymbol{u}_{S H}+\boldsymbol{u}_{S V} \\
& =A_{1} \boldsymbol{d} \exp \left(i \kappa_{P} \boldsymbol{d} \cdot \boldsymbol{x}\right)+A_{2} \boldsymbol{d}^{\perp} \exp \left(i \kappa_{S} \boldsymbol{d} \cdot \boldsymbol{x}\right)+A_{3} \boldsymbol{d} \times \boldsymbol{d}^{\perp} \exp \left(i \kappa_{S} \boldsymbol{d} \cdot \boldsymbol{x}\right)
\end{aligned}
$$

where the incident angle is chosen $\boldsymbol{d}=(-0.6838,0.4558,0.5698)$ and is not one of the directions for our plane wave basis. The amplitudes $A_{1}=A_{2}=A_{3}=1$. Especially, $\boldsymbol{d} \perp \boldsymbol{d}^{\perp} \perp\left(\boldsymbol{d} \times \boldsymbol{d}^{\perp}\right)$ and $\nabla \times \boldsymbol{u}_{P}=0$ and $\nabla \cdot \boldsymbol{u}_{S H}=\nabla \cdot \boldsymbol{u}_{S V}=0$. 


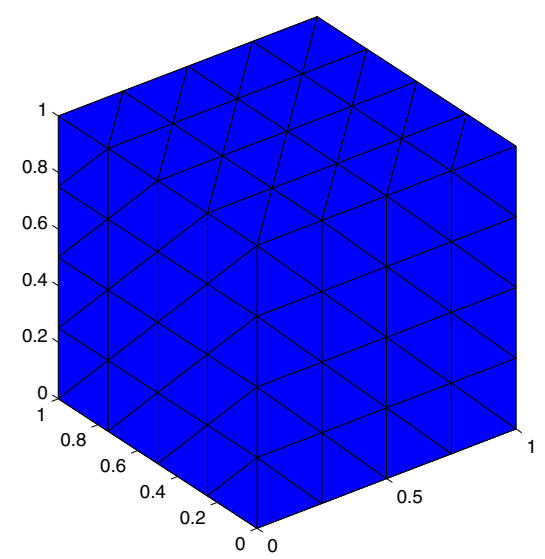

Figure 1. The third coarsest mesh used in simulations. The mesh size is $h=0.25$.

The traditional numerical flux $\eta$ of the elastic UWVF [16] extended to 3D is given, for a face with normal $\boldsymbol{n}$, by

$$
\eta=\omega \rho\left(c_{P} \boldsymbol{n} \otimes \boldsymbol{n}+c_{S}\left(\boldsymbol{s}_{1} \otimes \boldsymbol{s}_{1}+\boldsymbol{s}_{2} \otimes \boldsymbol{s}_{2}\right)\right)
$$

where $\otimes$ is defined as $\boldsymbol{n} \otimes \boldsymbol{n}=\boldsymbol{n} \boldsymbol{n}^{T}$, similarly for $\boldsymbol{s}_{1} \otimes \boldsymbol{s}_{1}$ and $\boldsymbol{s}_{2} \otimes \boldsymbol{s}_{2}$. The vector $\boldsymbol{s}_{1}$ is a polarization satisfying $\left|\boldsymbol{s}_{1}\right|=1$ and $\boldsymbol{s}_{1} \perp \boldsymbol{n}$, and the vector $\boldsymbol{s}_{2}$ is defined by $\boldsymbol{s}_{2}=\boldsymbol{n} \times \boldsymbol{s}_{1}$. Note that the numerical flux in [21] is chosen to be

$$
\eta=\omega \rho \Re\left\{c_{P}\right\} I
$$

where $I$ is an identity matrix, and $\Re\left\{c_{P}\right\}$ gives the real part of $c_{P}$. Note that in both cases $\eta$ is invertible, positive definite, and $\|\eta\|_{\infty}=\mathcal{O}(\omega),\left\|\eta^{-1}\right\|_{\infty}=\mathcal{O}\left(\omega^{-1}\right)$, as required in our theory.

First we choose the physical parameters as follows: frequency $f=0.8 \times 10^{4}$, angular frequency $\omega=2 \pi f=$ $5.0265 \times 10^{4}$, Young's modulus $E=200 \times 10^{9}$, Poisson ratio $\nu=0.3$ and density $\rho=7800$. Hence, $c_{P}=$ $5.8751 \times 10^{3}, c_{S}=3.1404 \times 10^{3}, \kappa_{P}=8.5557$ and $\kappa_{S}=16.0062$. We investigate how the different ratios of $p^{P}$ and $p^{S}$ affect the accuracy and conditioning, see also [16]. The mesh used in $p$-convergence computations is shown in Figure 1. The relative errors (discrete $\ell^{2}$-norm errors) are computed, in the $x=0.3, y=0.3$ and $z=0.3$ planes with a dense point grid $(201 \times 201$ points $)$. Denoting the $\ell^{2}$-norm on the plane $x=0.3$ by $\|\cdot\|_{\ell^{2}(x=0.3)}$ (similarly for the other planes), the relative error is computed as

$$
\operatorname{error}(\%)=\frac{1}{3}\left[\frac{\left\|\boldsymbol{u}-\boldsymbol{u}_{h}\right\|_{\ell^{2}(x=0.3)}}{\|\boldsymbol{u}\|_{\ell^{2}(x=0.3)}}+\frac{\left\|\boldsymbol{u}-\boldsymbol{u}_{h}\right\|_{\ell^{2}(y=0.3)}}{\|\boldsymbol{u}\|_{\ell^{2}(y=0.3)}}+\frac{\left\|\boldsymbol{u}-\boldsymbol{u}_{h}\right\|_{\ell^{2}(z=0.3)}}{\|\boldsymbol{u}\|_{\ell^{2}(z=0.3)}}\right] \times 100 \%
$$

where $\boldsymbol{u}$ is the exact solution and $\boldsymbol{u}_{h}$ is the UWVF solution. Note that our theory predicts error estimates in the global $\mathrm{L}^{2}$-norm, and not the norm in (4.3). We use this norm to investigate if it might be possible to prove estimates in norms other than the global $\mathrm{L}^{2}$-norm. Results are shown in Figure 2.

A critical issue with the UWVF is the conditioning of the matrix $D$. Results in Figure 2 show that the rate of increase of the condition number with respect to the number of unknowns per element increases as the ratio $p^{P} / p^{S}$ approaches 1 . This is reasonable since $\kappa_{P} \neq \kappa_{S}$ and is expected based on the results in [16, 21]. The errors are almost the same with different ratios of number of basis functions. We also present the computed error in Figure 2. When $p^{P}=p^{S}=16$ one of the directions in the basis is rather close to the incident direction that may explain the rapid decrease in the error at first. 

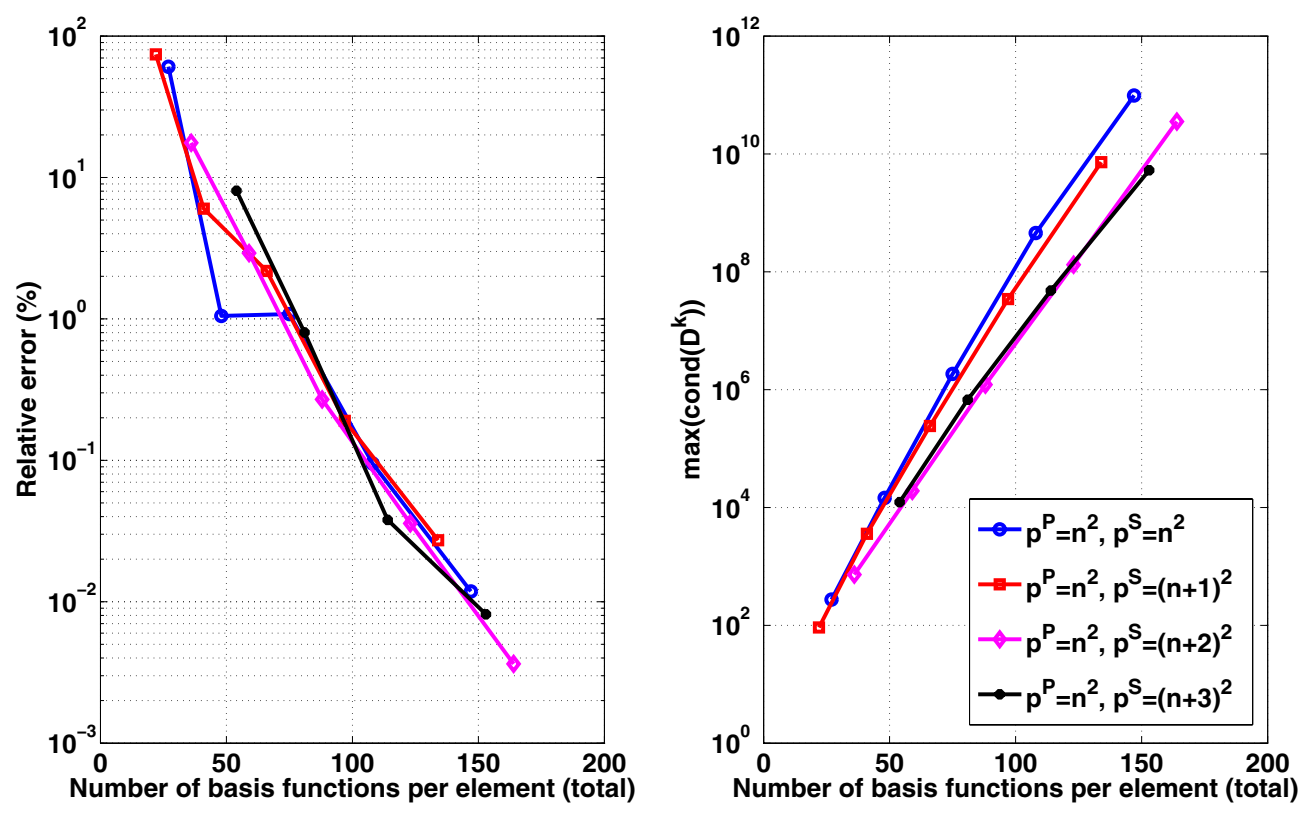

Figure 2. Results for $p$-convergence when $\kappa_{P}=8.5557$ and $\kappa_{S}=16.0062$. On the $x$-axis is shown the total number of basis functions per element i.e. $p^{P}+2 p^{S}$. On the left is shown relative error versus the total number of basis functions per element. On the right is shown the maximum of the condition number of the blocks $D^{k}$ versus the total number of basis functions per element. The ratios between the number of basis functions are $p^{P} / p^{S}=1, p^{P} / p^{S}=n^{2} /(n+1)^{2}$, $p^{P} / p^{S}=n^{2} /(n+2)^{2}$ and $p^{P} / p^{S}=n^{2} /(n+3)^{2}$ where $n \in \mathbb{N}$.

TABLE 1. Results for $h$-convergence when $\kappa_{P}=12.8335$ and $\kappa_{S}=24.0093$. The number of basis functions $p^{P}=36$ and $p^{S}=49$ (i.e. $q_{P}=5$ and $q_{S}=6$ ). The order of convergence of error is denoted by Order(error) and the growth rate of the condition number is denoted by $\operatorname{Order}($ cond).

\begin{tabular}{|c|c|c|c|c|}
\hline$h$ & Error(\%) & Order(error) & $\operatorname{Max}\left(\operatorname{cond}\left(D^{k}\right)\right)$ & Order(cond) \\
\hline 1.0 & 54.1824 & - & 136.6296 & - \\
\hline 0.5 & 25.1457 & 1.1075 & $1.4660 \mathrm{e} 4$ & -6.7455 \\
\hline 0.25 & 0.3063 & 6.3592 & $6.6385 \mathrm{e} 7$ & -12.1448 \\
\hline 0.125 & 0.0073 & 5.3909 & $1.7419 \mathrm{e} 11$ & -11.3575 \\
\hline
\end{tabular}

We investigate the $h$-convergence with $f=1.2 \times 10^{4}, \omega=2 \pi f=7.5398 \times 10^{4}, E=200 \times 10^{9}, \nu=0.3$, $\rho=7800, \kappa_{P}=12.8335$ and $\kappa_{S}=24.0093$ using $p^{P}=36$ and $p^{S}=49$ (i.e. $q_{P}=5$ and $q_{S}=6$ ). Since $q_{P} \geq 2 r_{P}+1$ and $q_{S} \geq 2 r_{S}+1$ we have $r_{P} \leq 2$ and $r_{S} \leq 5 / 2$ (i.e. $r_{S} \leq 2$ since $\left.r_{S} \in \mathbb{N}\right)$. The incident direction is $\boldsymbol{d}=(-0.6838,0.4558,0.5698)$ as previously. Results are shown in Table 1: the convergence rate for error (denoted Order(error)) and growth rate for the condition number (denoted Order(cond)).

Results in Table 1 show that the order of error convergence is approximately 5 or 6 (when the mesh size is adequately small). Similarly, the order of growth in the condition number is approximately -11 or -12 . Notice that the condition number is rather high when the mesh size is $h=0.125$. Results shown in Table 1 suggest that our theoretical error estimates are pessimistic since the order of convergence of error is closer to $q_{S}$ (or $q_{P}$ ) 
TABLE 2. Results for $h$-convergence when $\kappa_{P}=8.5557$ and $\kappa_{S}=16.0062$. The number of basis functions $p^{P}=9$ and $p^{S}=25$, i.e. $q_{P}=2$ and $q_{S}=4$. The order of convergence of error is denoted by Order(error) and the growth rate of the condition number is denoted by Order(cond).

\begin{tabular}{|c|c|c|c|c|}
\hline$h$ & Error(\%) & Order(error) & $\operatorname{Max}\left(\operatorname{cond}\left(D^{k}\right)\right)$ & Order(cond) \\
\hline 1.0 & 90.6057 & - & 20.5633 & - \\
\hline 0.5 & 38.8714 & 1.2209 & 200.2592 & -3.3197 \\
\hline 0.25 & 2.9209 & 3.7342 & $1.9154 \mathrm{e} 4$ & -6.5796 \\
\hline 0.125 & 0.1716 & 4.0893 & $2.0054 \mathrm{e} 6$ & -6.7101 \\
\hline 0.0625 & 0.0152 & 3.4969 & $1.5940 \mathrm{e} 8$ & -6.3126 \\
\hline
\end{tabular}

TABLE 3. Results for $h$-convergence when $\kappa_{P}=6.4168$ and $\kappa_{S}=12.0047$. The number of basis functions $p^{P}=9$ and $p^{S}=16$, i.e. $q_{P}=2$ and $q_{S}=3$. The order of convergence of error is denoted by Order(error) and the growth rate of the condition number is denoted by $\operatorname{Order}(\mathrm{cond})$.

\begin{tabular}{|c|c|c|c|c|}
\hline$h$ & Error(\%) & Order(error) & $\operatorname{Max}\left(\operatorname{cond}\left(D^{k}\right)\right)$ & Order(cond) \\
\hline 1.0 & 35.8301 & - & 30.3593 & - \\
\hline 0.5 & 13.4938 & 1.4089 & 378.3482 & -3.6395 \\
\hline 0.25 & 2.1239 & 2.6675 & $1.4863 \mathrm{e} 4$ & -5.2959 \\
\hline 0.125 & 0.2707 & 2.9719 & $3.6232 \mathrm{e} 5$ & -4.6075 \\
\hline 0.0625 & 0.0442 & 2.6146 & $6.8682 \mathrm{e} 6$ & -4.2446 \\
\hline
\end{tabular}

instead of $r_{S}\left(\right.$ or $\left.r_{P}\right)$. However, our error estimates use a vector and scalar potential to reduce the plane wave estimates to those for the Helmholtz problem. This likely reduces the predicted order of convergence. A direct approximation theory for elastic vector unknowns might improve the estimates (however, we currently have no way of doing this).

Now we want to investigate numerically cases in which our theory does not predict convergence. We again investigate $h$-convergence keeping the number of basis functions for $p^{P}$ and $p^{S}$ fixed. Results are shown in Table 2 for the case when $\kappa_{P}=8.5557, \kappa_{S}=16.0062$, and the number of basis functions are $p^{P}=9$ and $p^{S}=25$, i.e. $q_{P}=2$ and $q_{S}=4$. Since $q_{P} \geq 2 r_{P}+1$ and $q_{S} \geq 2 r_{S}+1$ we have $r_{P} \leq 1 / 2$ (i.e. $r_{P}=0$ since $r_{P} \in \mathbb{N}$ ) and $r_{S} \leq 3 / 2$ (i.e. $r_{S} \leq 1$ since $r_{S} \in \mathbb{N}$ ). In addition, in Table 3 is shown results using lower wave numbers than in Table 2. We choose $f=0.6 \times 10^{4}, E=200 \times 10^{9}, \nu=0.3$ and $\rho=7800$ then $\kappa_{P}=6.4168$ and $\kappa_{S}=12.0047$. The number of basis functions are chosen as $p^{P}=9$ and $p^{S}=16$, i.e. $q_{P}=2$ and $q_{S}=3$ and $r_{P}=0$ and $r_{S} \leq 1$.

Results shown in Table 2 imply that the order of convergence for the error is roughly 4 when $p^{P}=9$ and $p^{S}=25$ (i.e. $r_{P}=0$ and $r_{S} \leq 1$ ). Similarly than in Table 1 the order of convergence of error is closer the value $q_{S}=4\left(\right.$ or $\left.q_{P}=3\right)$ than $r_{S} \leq 1$ (or $\left.r_{P}=0\right)$. The order of condition number increase is roughly between -6 and -7 when $p^{P}=9$ and $p^{S}=25$ when the element size is small enough.

Results shown in Table 3 are similar to those in Table 2. In particular, the order of convergence for the error is roughly 3 (when the mesh is fine enough). The order of growth of the condition number is approximately -5 when $p^{P}=9$ and $p^{S}=16\left(r_{P}=0\right.$ and $\left.r_{S} \leq 1\right)$ and the mesh size is small enough. In Table 3 , cf. Tables 1 and 2 , the order of convergence of error is closer the value $q_{S}=3\left(\right.$ or $\left.q_{P}=2\right)$ than $r_{S}\left(\right.$ or $\left.r_{P}\right)$.

\section{Conclusions}

This paper is a continuation of [16] and is particularly motivated by the work in $[4,13,14,25,26]$. We have derived error estimates for the UWVF in 3D linear elasticity using discontinuous Galerkin techniques as in $[4,11,18]$. In particular we provide a final error estimate in the $\mathrm{L}^{2}(\Omega)$ norm taking into account that the 
number of basis functions for $\mathrm{P}$ - and $\mathrm{S}$-waves are different in practice. Numerical results show that the UWVF is a feasible method for solving wave problems in $3 \mathrm{D}$ linear elasticity, and suggest that the real convergence rates are closer to $q_{S}\left(\right.$ or $\left.q_{P}\right)$ instead of $r_{S}\left(\right.$ or $\left.r_{P}\right)$. Therefore, as we have discussed, our error estimate is pessimistic (similarly to the error estimates of the acoustic UWVF in [4]).

In future we shall demonstrate the UWVF for solving more complex and practical problems in linear elasticity including problem having surface waves and other rapidly decaying evanescent waves. In particular, we shall extent our 2D fluid-solid code to 3D fluid-solid interface problems using special choices of basis functions similar to those introduced in [22].

Acknowledgements. The authors would like to thank the anonymous reviewers for their careful and helpful reviews.

\section{REFERENCES}

[1] D.N. Arnold, F. Brezzi, B. Cockburn and L.D. Marini, Unified analysis of discontinuous Galerkin methods for elliptic problems. SIAM J. Numer. Anal. 39 (2002) 1749-1779.

[2] A.H. Barnett and T. Betcke, An exponentially convergent nonpolynomial finite element method for time-harmonic scattering from polygons. SIAM J. Sci. Comput. 32 (2010) 1417-1441.

[3] S.C. Brenner and L.R. Scott, The mathematical theory of finite element methods, 3rd edition. Springer (2008).

[4] A. Buffa and P. Monk, Error estimates for the ultra weak variational formulation of the Helmholtz equation. ESAIM: M2AN 42 (2008) 925-940.

[5] O. Cessenat, Application d'une nouvelle formulation variationnelle aux équations d'ondes harmoniques. Problèmes de Helmholtz 2D et de Maxwell 3D. Ph.D. thesis, Université Paris IX Dauphine (1996).

[6] O. Cessenat and B. Després, Application of an ultra weak variational formulation of elliptic PDEs to the two-dimensional Helmholtz problem. SIAM J. Numer. Anal. 35 (1998) 255-299.

[7] P. Cummings and X. Feng, Sharp regularity coefficient estimates for complex-valued acoustic and elastic Helmholtz equations. Math. Mod. Methods Appl. Sci. 16 (2006) 139-160.

[8] A. El Kacimi and O. Laghrouche, Numerical modeling of elastic wave scattering in frequency domain by partition of unity finite element method. Int. J. Numer. Methods Eng. 77 (2009) 1646-1669.

[9] C. Farhat, I. Harari and L.P. Franca, A discontinuous enrichment method. Comput. Methods Appl. Mech. Eng. 190 (2001) 6455-6479.

[10] C. Farhat, I. Harari and U. Hetmaniuk, A discontinuous Galerkin method with Lagrange multipliers for the solution of Helmholtz problems in the mid-frequency regime. Comput. Methods Appl. Mech. Eng. 192 (2003) 1389-1429.

[11] G. Gabard, Discontinuous Galerkin methods with plane waves for time-harmonic problems. J. Comput. Phys. 225 (2007) 1961-1984.

[12] R. Hardin, N. Sloane and W. Smith, Spherical coverings. Available on http://www.research.att.com/ ${ }^{\sim n j a s / c o v e r i n g s / ~}$ index.html (1994).

[13] R. Hiptmair, A. Moiola and I. Perugia, Plane wave discontinuous Galerkin methods for the 2D Helmholtz equation: analysis of the p-version. SIAM J. Numer. Anal. 49 (2011) 264-284.

[14] R. Hiptmair, A. Moiola and I. Perugia, Error analysis of Trefftz-discontinuous Galerkin methods for the time-harmonic Maxwell equations. Math. Comput. In press.

[15] T. Huttunen, P. Monk and J.P. Kaipio, Computational aspects of the ultra-weak variational formulation. J. Comput. Phys. 182 (2002) 27-46.

[16] T. Huttunen, P. Monk, F. Collino and J.P. Kaipio, The ultra weak variational formulation for elastic wave problems. SIAM J. Sci. Comput. 25 (2004) 1717-1742.

[17] T. Huttunen, P. Monk and J.P. Kaipio, The perfectly matched layer for the ultra weak variational formulation of the 3D Helmholtz equation. Int. J. Numer. Methods Eng. 61 (2004) 1072-1092.

[18] T. Huttunen, M. Malinen and P. Monk, Solving Maxwell's equations using the ultra weak variational formulation. J. Comput. Phys. 223 (2007) 731-758.

[19] T. Huttunen, J.P. Kaipio and P. Monk, An ultra-weak method for acoustic fluid-solid interaction. J. Comput. Appl. Math. 213 (2008) 1667-1685.

[20] V.D. Kupradze, Potential methods in the theory of elasticity. Israel Program for Scientific Translations (1965).

[21] T. Luostari, T. Huttunen and P. Monk, The ultra weak variational formulation for 3D elastic wave problems, in Proc. 20th International Congress on Acoustics, ICA (2010). Available in 2010 on http://www.acoustics.asn.au .

[22] P. Massimi, R. Tezaur and C. Farhat, A discontinuous enrichment method for three-dimensional multiscale harmonic wave propagation problems in multi-fluid and fluid-solid media. Int. J. Numer. Methods Eng. 76 (2008) 400-425.

[23] M.M. Melenk and I. Babuška, The partition of unity finite element method: basic theory and applications. Comput. Methods Appl. Mech. Eng. 139 (1996) 289-314.

[24] A. Moiola, Trefftz-Discontinuous Galerkin Methods for Time-Harmonic Wave Problems. Ph.D. thesis, ETH Zürich (2011). 
[25] A. Moiola, Plane wave approximation in linear elasticity. To appear in Appl. Anal.

[26] A. Moiola, R. Hiptmair and I. Perugia, Plane wave approximation of homogeneous Helmholtz solutions. Z. Angew. Math. Phys. 65 (2011) 809-837.

[27] P. Monk and D.-Q. Wang, A least squares method for the Helmholtz equation. Comput. Methods Appl. Mech. Eng. 175 (1999) $121-136$.

[28] Y.-H. Pao, Betti's identity and transition matrix for elastic waves. J. Acoust. Soc. Am. 64 (1978) 302-310.

[29] E. Perrey-Debain, Plane wave decomposition in the unit disc: convergence estimates and computational aspects. J. Comput. Appl. Math. 193 (2006) 140-156.

[30] I. Sloan and R. Womersley, Extremal systems of points and numerical integration on the sphere. Adv. Comput. Math. 21 (2004) 107-125.

[31] D. Wang, J. Toivanen, R. Tezaur and C. Farhat, Overview of the discontinuous enrichment method, the ultra-weak variational formulation, and the partition of unity method for acoustic scattering in the medium frequency regime and performance comparisons. Int. J. Numer. Methods Eng. 89 (2012) 403-417.

[32] R. Womersley and I. Sloan, Interpolation and cubature on the sphere. Available on http://web.maths.unsw.edu.au/ ${ }^{\sim} \mathrm{rsw} /$ Sphere. 